\title{
Tamarkin's construction is equivariant with respect to the action of the Grothendieck-Teichmueller group
}

\author{
Vasily Dolgushev $^{1}$ • Brian Paljug ${ }^{1}$
}

Received: 3 April 2015 / Accepted: 10 June 2015 / Published online: 25 June 2015

(C) Tbilisi Centre for Mathematical Sciences 2015

\begin{abstract}
Recall that Tamarkin's construction (Hinich, Forum Math 15(4):591-614, 2003, arXiv:math.QA/0003052; Tamarkin, 1998, arXiv:math/9803025) gives us a map from the set of Drinfeld associators to the set of homotopy classes of $L_{\infty}$ quasi-isomorphisms for Hochschild cochains of a polynomial algebra. Due to results of Drinfeld (Algebra i Analiz 2(4):149-181, 1990) and Willwacher Invent Math 200(3):671-760, 2015 both the source and the target of this map are equipped with natural actions of the Grothendieck-Teichmueller group GRT 1 . In this paper, we use the result from Paljug (JHRS, 2015, arXiv:1305.4699) to prove that this map from the set of Drinfeld associators to the set of homotopy classes of $L_{\infty}$ quasi-isomorphisms for Hochschild cochains is $\mathrm{GRT}_{1}$-equivariant.
\end{abstract}

Keywords Formality theorems · Algebraic operads · Associators

\section{Contents}

1 Introduction . . . . . . . . . . . . . . . . . . . . . . . 504

1.1 Notation and conventions . . . . . . . . . . . . . . . . . . . . 505

$1.1 .1 \mathrm{Ger}_{\infty}$-algebras and a basis in $\operatorname{Ger}^{\vee}(n) \ldots \ldots . \ldots$. . . . . . . . . . . 507

1.1.2 The dg operad Braces . . . . . . . . . . . . . . . . . . . . 508

2 Tamarkin's construction in a nutshell . . . . . . . . . . . . . . . . . . . 510

3 Actions of $\mathrm{GRT}_{1} \ldots \ldots \ldots \ldots \ldots \ldots \ldots \ldots \ldots$

Communicated by Jim Stasheff.

$\triangle$ Vasily Dolgushev vald@temple.edu

Brian Paljug

brian.paljug@temple.edu

1 Department of Mathematics, Temple University, Wachman Hall Rm. 638, 1805 N. Broad St., Philadelphia, PA 19122, USA 


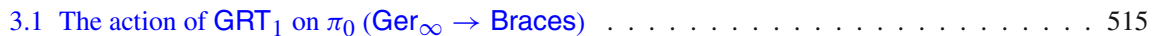

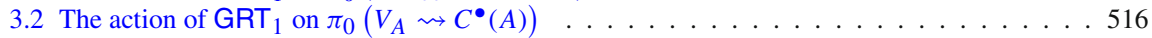

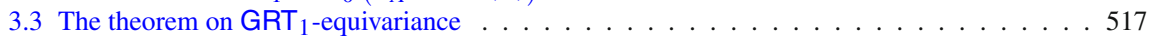

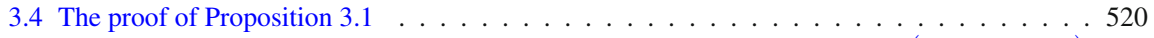

4 Final remarks: connecting Drinfeld associators to the set of homotopy classes $\pi_{0}\left(V_{A} \rightsquigarrow C^{\bullet}(A)\right) 523$

4.1 The sets DrAssoc ${ }_{\kappa}$ of Drinfeld associators . . . . . . . . . . . . . . . 523

4.2 A map $\mathfrak{B}$ from DrAssoc $_{1}$ to $\pi_{0}\left(\operatorname{Ger}_{\infty} \rightarrow\right.$ Braces $) \ldots \ldots \ldots . \ldots . . \ldots 24$

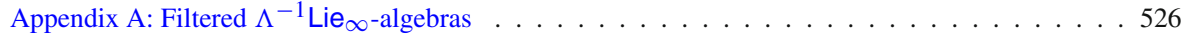

A.1: A lemma on adjusting Maurer-Cartan elements . . . . . . . . . . . . . . . . 528

A.2: Convolution $\Lambda^{-1} \mathrm{Lie}_{\infty}$-algebra, $\infty$-morphisms and their homotopies $\ldots \ldots \ldots 30$

Appendix B: Tamarkin's rigidity . . . . . . . . . . . . . . . . . . . 532

B.1: The standard Gerstenhaber structure on $V_{A}$ is "rigid" . . . . . . . . . . . . . 537

B.2 The Gerstenhaber algebra $V_{A}$ is intrinsically formal . . . . . . . . . . . . . 541

Appendix C: On derivations of $\mathrm{Cyl}\left(\Lambda^{2} \mathrm{coCom}\right) \ldots \ldots \ldots \ldots \ldots$

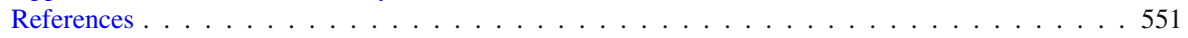

\section{Introduction}

Let $\mathbb{K}$ be a field of characteristic zero, $A=\mathbb{K}\left[x^{1}, x^{2}, \ldots, x^{d}\right]$ be the algebra of functions on the affine space $\mathbb{K}^{d}$, and $V_{A}$ be the algebra of polyvector fields on $\mathbb{K}^{d}$. Let us recall that Tamarkin's construction [15,24] gives us a map from the set of Drinfeld associators to the set of homotopy classes of $L_{\infty}$ quasi-isomorphisms from $V_{A}$ to the Hochschild cochain complex $C^{\bullet}(A):=C^{\bullet}(A, A)$ of $A$.

In paper [27], among proving many other things, Thomas Willwacher constructed a

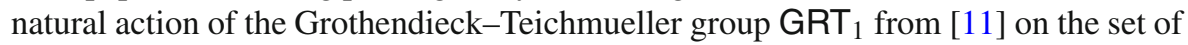
homotopy classes of $L_{\infty}$ quasi-isomorphisms from $V_{A}$ to $C^{\bullet}(A)$. On the other hand, it is known [11] that the group GRT 1 acts simply transitively on the set of Drinfeld associators.

The goal of this paper is to prove $\mathrm{GRT}_{1}$-equivariance of the map resulting from Tamarkin's construction using Theorem 4.3 from [22]. We should remark that the statement about GRT 1 -equivariance of Tamarkin's construction was made in [27] (see the last sentence of Sect. 10.2 in [27, Version 3]) in which the author stated that "it is easy to see". The modest goal of this paper is to convince the reader that this statement can indeed be proved easily. However, the proof requires an additional tool developed in [22].

In this paper, we also prove various statements related to Tamarkin's construction $[15,24]$ which are "known to specialists" but not proved in the literature in the desired generality. In fact, even the formulation of the problem of $\mathrm{GRT}_{1}$-equivariance of Tamarkin's construction requires some additional work.

In this paper, Tamarkin's construction is presented in the slightly more general setting of graded affine space versus the particular case of the usual affine space. Thus, $A$ is always the free (graded) commutative algebra over $\mathbb{K}$ in variables $x^{1}, x^{2}, \ldots, x^{d}$ of (not necessarily zero) degrees $t_{1}, t_{2}, \ldots, t_{d}$, respectively. Furthermore, $V_{A}$ denotes the Gerstenhaber algebra of polyvector fields on the corresponding graded affine space, i.e.

$$
V_{A}:=S_{A}\left(\mathbf{s} \operatorname{Der}_{\mathbb{K}}(A)\right),
$$

where $\operatorname{Der}_{\mathbb{K}}(A)$ denotes the $A$-module of derivations of $A, \mathbf{s}$ is the operator which shifts the degree up by 1 , and $S_{A}(M)$ denotes the free (graded) commutative algebra on the $A$-module $M$. 
The paper is organized as follows. In Sect. 2, we briefly review the main part of Tamarkin's construction and prove that it gives us a map $\mathfrak{T}$ [see Eq. (2.20)] from the set of homotopy classes of certain quasi-isomorphisms of $\mathrm{dg}$ operads to the set of homotopy classes of $L_{\infty}$ quasi-isomorphisms for Hochschild cochains of $A$.

In Sect. 3, we introduce a (prounipotent) group which is isomorphic (due to Willwacher's theorem [27, Theorem 1.2]) to the prounipotent part GRT 1 of the Grothendieck-Teichmueller group GRT introduced in [11] by Drinfeld. We recall from [27] the actions of the group (isomorphic to $\mathrm{GRT}_{1}$ ) both on the source and the target of the map $\mathfrak{T}$ (2.20). Finally, we prove the main result of this paper (see Theorem 3.3) which says that Tamarkin's map $\mathfrak{T}$ [see Eq. (2.20)] is GRT ${ }_{1}$-equivariant.

In Sect. 4, we recall how to use the map $\mathfrak{T}$ [see Eq. (2.20) from Sect. 2], a specific solution of Deligne's conjecture on the Hochschild complex, and the formality of the operad of little discs [25] to construct a map from the set of Drinfeld associators to the set of homotopy classes of $L_{\infty}$ quasi-isomorphisms for Hochschild cochains of $A$. Finally, we deduce, from Theorem 3.3, GRT $_{1}$-equivariance of the resulting map from the set of Drinfeld associators. The latter statement (see Corollary 4.1 in Sect. 4) can be deduced from what is written in [27] and Theorem 3.3 given in Sect. 3. However, we decided to add Sect. 4 just to make the story more complete.

Appendices, at the end of the paper, are devoted to proofs of various technical statements used in the body of the paper.

Remark 1.1 While this paper was in preparation, the 4-th version of preprint [27] appeared on arXiv.org. In Remark 10.1 of [27, Version 4], Willwacher gave a sketch of admittedly more economic proof of equivariance of Tamarkin's construction with respect to the action of $\mathrm{GRT}_{1}$.

\subsection{Notation and conventions}

The ground field $\mathbb{K}$ has characteristic zero. For most of algebraic structures considered here, the underlying symmetric monoidal category is the category $\mathrm{Ch}_{\mathbb{K}}$ of unbounded cochain complexes of $\mathbb{K}$-vector spaces. We will frequently use the ubiquitous combination "dg" (differential graded) to refer to algebraic objects in $\mathrm{Ch}_{\mathbb{K}}$. For a cochain complex $V$ we denote by $\mathbf{s} V$ (resp. by $\mathbf{s}^{-1} V$ ) the suspension (resp. the desuspension) of $V$. In other words,

$$
(\mathbf{s} V)^{\bullet}=V^{\bullet-1}, \quad\left(\mathbf{s}^{-1} V\right)^{\bullet}=V^{\bullet+1} .
$$

Any $\mathbb{Z}$-graded vector space $V$ is tacitly considered as the cochain complex with the zero differential. For a homogeneous vector $v$ in a cochain complex or a graded vector space the notation $|v|$ is reserved for its degree.

The notation $S_{n}$ is reserved for the symmetric group on $n$ letters and $\mathrm{Sh}_{p_{1}, \ldots, p_{k}}$ denotes the subset of $\left(p_{1}, \ldots, p_{k}\right)$-shuffles in $S_{n}$, i.e. $\mathrm{Sh}_{p_{1}, \ldots, p_{k}}$ consists of elements $\sigma \in S_{n}, n=p_{1}+p_{2}+\cdots+p_{k}$ such that

$$
\begin{gathered}
\sigma(1)<\sigma(2)<\cdots<\sigma\left(p_{1}\right), \\
\sigma\left(p_{1}+1\right)<\sigma\left(p_{1}+2\right)<\cdots<\sigma\left(p_{1}+p_{2}\right), \\
\cdots \\
\sigma\left(n-p_{k}+1\right)<\sigma\left(n-p_{k}+2\right)<\cdots<\sigma(n) .
\end{gathered}
$$


We tacitly assume the Koszul sign rule. In particular,

$$
(-1)^{\varepsilon\left(\sigma ; v_{1}, \ldots, v_{m}\right)}
$$

will always denote the sign factor corresponding to the permutation $\sigma \in S_{m}$ of homogeneous vectors $v_{1}, v_{2}, \ldots, v_{m}$. Namely,

$$
(-1)^{\varepsilon\left(\sigma ; v_{1}, \ldots, v_{m}\right)}:=\prod_{(i<j)}(-1)^{\left|v_{i}\right|\left|v_{j}\right|},
$$

where the product is taken over all inversions $(i<j)$ of $\sigma \in S_{m}$.

For a pair $V, W$ of $\mathbb{Z}$-graded vector spaces we denote by

$$
\operatorname{Hom}(V, W)
$$

the corresponding inner-hom object in the category of $\mathbb{Z}$-graded vector spaces, i.e.

$$
\operatorname{Hom}(V, W):=\bigoplus_{m} \operatorname{Hom}_{\mathbb{K}}^{m}(V, W)
$$

where $\operatorname{Hom}_{\mathbb{K}}^{m}(V, W)$ consists of $\mathbb{K}$-linear maps $f: V \rightarrow W$ such that

$$
f\left(V^{\bullet}\right) \subset W^{\bullet+m} .
$$

For a commutative algebra $B$ and a $B$-module $M$, the notation $S_{B}(M)\left(\operatorname{resp} . \underline{S}_{B}(M)\right)$ is reserved for the symmetric $B$-algebra (resp. the truncated symmetric $B$-algebra) on $M$, i.e.

$$
S_{B}(M):=B \oplus M \oplus S_{B}^{2}(M) \oplus S_{B}^{3}(M) \oplus \cdots,
$$

and

$$
\underline{S}_{B}(M):=M \oplus S_{B}^{2}(M) \oplus S_{B}^{3}(M) \oplus \cdots
$$

For an $A_{\infty}$-algebra $\mathcal{A}$, the notation $C^{\bullet}(\mathcal{A})$ is reserved for the Hochschild cochain complex of $\mathcal{A}$ with coefficients in $\mathcal{A}$.

We denote by Com (resp. Lie, Ger) the operad governing commutative (and associative) algebras without unit (resp. the operad governing Lie algebras, Gerstenhaber algebras ${ }^{1}$ without unit). Furthermore, we denote by coCom the cooperad which is obtained from Com by taking the linear dual. The coalgebras over coCom are cocommutative (and coassociative) coalgebras without counit.

The notation Cobar is reserved for the cobar construction [5, Section 3.7].

For an operad (resp. a cooperad) $P$ and a cochain complex $V$ we denote by $P(V)$ the free $P$-algebra (resp. the cofree ${ }^{2} P$-coalgebra) generated by $V$ :

\footnotetext{
1 See, for example, Appendix A in [9].

2 We tacitly assume that all coalgebras are nilpotent.
} 


$$
P(V):=\bigoplus_{n \geq 0}\left(P(n) \otimes V^{\otimes n}\right)_{S_{n}} .
$$

For example,

$$
\operatorname{Com}(V)=\operatorname{coCom}(V)=\underline{S}(V) .
$$

We denote by $\Lambda$ the underlying collection of the endomorphism operad

$$
\mathrm{End}_{\mathbf{s} \mathbb{K}}
$$

of the one-dimensional space $\mathbf{s} \mathbb{K}$ placed in degree 1 . The $n$-the space of $\Lambda$ is

$$
\Lambda(n)=\operatorname{sgn}_{n} \otimes \mathbf{s}^{1-n},
$$

where $\operatorname{sgn}_{n}$ denotes the sign representation of the symmetric group $S_{n}$. Recall that $\Lambda$ is naturally an operad and a cooperad.

For a (co)operad $P$, we denote by $\Lambda P$ the (co)operad which is obtained from $P$ by tensoring with $\Lambda$ :

$$
\Lambda P:=\Lambda \otimes P .
$$

It is clear that tensoring with

$$
\Lambda^{-1}:=\operatorname{End}_{\mathbf{s}^{-1}} \mathbb{K}
$$

gives us the inverse of the operation $P \mapsto \Lambda P$.

For example, the dg operad Cobar $\left(\Lambda\right.$ coCom) governs $L_{\infty}$-algebras and the dg operad

$$
\operatorname{Cobar}\left(\Lambda^{2} \text { coCom }\right)
$$

governs $\Lambda$ Lie $_{\infty}$-algebras.

\subsection{1 $\mathrm{Ger}_{\infty}$-algebras and a basis in $\operatorname{Ger}^{\vee}(n)$}

Let us recall that $\mathrm{Ger}_{\infty}$-algebras (or homotopy Gerstenhaber algebras) are governed by the dg operad

$$
\operatorname{Cobar}\left(\operatorname{Ger}^{\vee}\right),
$$

where Ger ${ }^{\vee}$ is the cooperad which is obtained by taking the linear dual of $\Lambda^{-2}$ Ger.

For our purposes, it is convenient to introduce the free $\Lambda^{-2}$ Ger-algebra $\Lambda^{-2} \operatorname{Ger}\left(b_{1}, b_{2}, \ldots, b_{n}\right)$ in $n$ auxiliary variables $b_{1}, b_{2}, \ldots, b_{n}$ of degree 0 and identify the $n$-th space $\Lambda^{-2} \operatorname{Ger}(n)$ of $\Lambda^{-2} \operatorname{Ger}$ with the subspace of $\Lambda^{-2} \operatorname{Ger}\left(b_{1}, b_{2}, \ldots\right.$, $b_{n}$ ) spanned by $\Lambda^{-2}$ Ger-monomials in which each variable $b_{j}$ appears exactly once. For example, $\Lambda^{-2} \operatorname{Ger}(2)$ is spanned by the monomials $b_{1} b_{2}$ and $\left\{b_{1}, b_{2}\right\}$ of degrees 2 and 1 , respectively. 
Let us consider the ordered partitions of the set $\{1,2, \ldots, n\}$

$$
\left\{i_{11}, i_{12}, \ldots, i_{1 p_{1}}\right\} \sqcup\left\{i_{21}, i_{22}, \ldots, i_{2 p_{2}}\right\} \sqcup \cdots \sqcup\left\{i_{t 1}, i_{t 2}, \ldots, i_{t p_{t}}\right\}
$$

satisfying the following properties:

- for each $1 \leq \beta \leq t$ the index $i_{\beta p_{\beta}}$ is the biggest among $i_{\beta 1}, \ldots, i_{\beta p_{\beta}}$

- $i_{1 p_{1}}<i_{2 p_{2}}<\cdots<i_{t p_{t}}$ (in particular, $i_{t p_{t}}=n$ ).

It is clear that the monomials

$$
\left\{b_{i_{11}}, \ldots,\left\{b_{i_{1\left(p_{1}-1\right)}}, b_{i_{1 p_{1}}}\right\} .\right\} \ldots\left\{b_{i_{t 1}}, \ldots,\left\{b_{i_{t\left(p_{t}-1\right)}}, b_{i_{t p_{t}}}\right\} .\right\}
$$

corresponding to all ordered partitions (1.6) satisfying the above properties form a basis of the space $\Lambda^{-2} \operatorname{Ger}(n)$.

In this paper, we use the notation

$$
\left(\left\{b_{i_{11}}, \ldots,\left\{b_{i_{1\left(p_{1}-1\right)}}, b_{i_{1 p_{1}}}\right\} . . .\left\{\left\{b_{i_{t 1}}, \ldots,\left\{b_{i_{t\left(p_{t}-1\right)}}, b_{i_{t p_{t}}}\right\} . .\right\}\right)^{*}\right.\right.
$$

for the elements of the dual basis in $\operatorname{Ger}^{\vee}(n)=\left(\Lambda^{-2} \operatorname{Ger}(n)\right)^{*}$.

\subsubsection{The dg operad Braces}

In this brief subsection, we recall the dg operad Braces from [9, Section 9] and [17]. ${ }^{3}$

Following [9], we introduce, for every $n \geq 1$, the auxiliary set $\mathcal{T}(n)$. An element of $\mathcal{T}(n)$ is a planted ${ }^{4}$ planar tree $T$ with the following data

- a partition of the set $V(T)$ of vertices

$$
V(T)=V_{\mathrm{lab}}(T) \sqcup V_{\nu}(T) \sqcup V_{\text {root }}(T)
$$

into the singleton $V_{\text {root }}(T)$ consisting of the root vertex, the set $V_{\text {lab }}(T)$ consisting of $n$ vertices which we call labeled, and the set $V_{v}(T)$ consisting of vertices which we call neutral;

- a bijection between the set $V_{\text {lab }}(T)$ and the set $\{1,2, \ldots, n\}$.

We require that each element $T$ of $\mathcal{T}(n)$ satisfies this condition.

Condition 1.2 Every neutral vertex of T has at least 2 incoming edges.

Elements of $\mathcal{T}(n)$ are called brace trees.

For $n \geq 1$, the vector space Braces $(n)$ consists of all finite linear combinations of brace trees in $\mathcal{T}(n)$. To define a structure of a graded vector space on $\operatorname{Braces}(n)$, we declare that each brace tree $T \in \mathcal{T}(n)$ carries degree

$$
|T|=2\left|V_{v}(T)\right|-|E(T)|+1,
$$

where $\left|V_{v}(T)\right|$ denotes the total number of neutral vertices of $T$ and $|E(T)|$ denotes the total number of edges of $T$.

\footnotetext{
3 In paper [17], the dg operad Braces is called the "minimal operad".

4 Recall that a planted tree is a rooted tree whose root vertex has valency 1.
} 
Fig. 1 A brace tree $T \in \mathcal{T}(2)$

Fig. 2 A brace tree $T_{21} \in \mathcal{T}(2)$

Fig. 3 A brace tree $T \cup \in \mathcal{T}(2)$

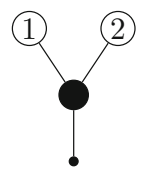

Fig. 4 A brace tree

$T_{\cup \text { opp }} \in \mathcal{T}(2)$

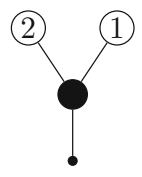

Fig. 5 The brace tree

$T_{\mathrm{id}} \in \mathcal{T}(1)$

Examples of brace trees in $\mathcal{T}(2)$ (and hence vectors in Braces(2)) are shown on Figs. 1, 2, 3 and 4.

According to (1.9), the brace trees $T$ and $T_{21}$ on Figs. 1 and 2, respectively, carry degree -1 and the brace trees $T \cup, T_{\cup}$ opp on Figs. 3 and 4, respectively, carry degree 0 .

Condition 1.2 implies that $\mathcal{T}(1)$ consists of exactly one brace tree $T_{\text {id }}$ shown on Fig. 5.

Hence we have Braces $(1)=\mathbb{K}$.

Finally, we set Braces $(0)=\mathbf{0}$.

For the definition of the operadic multiplications on Braces, we refer the reader to $^{5}[9$, Section 8] and, in particular, Example 8.2. For the definition of the differential on Braces, we refer the reader to [9, Section 8.1] and, in particular, Example 8.4.

Let us also recall that the dg operad Braces acts naturally on the Hochschild cochain complex $C^{\bullet}(\mathcal{A})$ of any $A_{\infty}$-algebra $\mathcal{A}$. For example, if $T$ (resp. $T_{21}$ ) is the brace tree shown on Fig. 1 (resp. Fig. 2), then the expression

$$
T\left(P_{1}, P_{2}\right)+T_{21}\left(P_{1}, P_{2}\right), \quad P_{1}, P_{2} \in C^{\bullet}(\mathcal{A})
$$

coincides (up to a sign factor) with the Gerstenhaber bracket of $P_{1}$ and $P_{2}$. Similarly, if $T \cup$ is the brace tree shown on Fig. 3, then the expression

\footnotetext{
5 Strictly speaking Braces is a suboperad of the dg operad defined in [9, Section 8].
} 


$$
T_{\cup}\left(P_{1}, P_{2}\right), \quad P_{1}, P_{2} \in C^{\bullet}(\mathcal{A})
$$

coincides (up to a sign factor) with the cup product of $P_{1}$ and $P_{2}$.

For the precise construction of the action of Braces on $C^{\bullet}(\mathcal{A})$, we refer the reader to [9, Appendix B].

\section{Tamarkin's construction in a nutshell}

Various solutions of Deligne's conjecture on the Hochschild cochain complex [3,4,8, $17,21,23,26]$ imply that the $\mathrm{dg}$ operad Braces is quasi-isomorphic to the dg operad

$$
C_{-\bullet}\left(E_{2}, \mathbb{K}\right)
$$

of singular chains for the little disc operad $E_{2}$.

Combining this statement with the formality $[18,25]$ for the $\operatorname{dg}$ operad $C_{-\bullet}\left(E_{2}, \mathbb{K}\right)$, we conclude that the dg operad Braces is quasi-isomorphic to the operad Ger. Hence there exists a quasi-isomorphism of $\mathrm{dg}$ operads

$$
\Psi: \operatorname{Ger}_{\infty} \rightarrow \text { Braces }
$$

for which the vector ${ }^{6} \Psi\left(\mathbf{s}\left(b_{1} b_{2}\right)^{*}\right)$ is cohomologous to the sum $T+T_{21}$ and the vector $\Psi\left(\mathbf{s}\left\{b_{1}, b_{2}\right\}^{*}\right)$ is cohomologous to

$$
\frac{1}{2}\left(T_{\cup}+T_{\cup o p p}\right)
$$

where $T$ (resp. $T_{21}, T_{\cup}, T_{\cup}$ opp ) is the brace tree depicted on Fig. 1 (resp. Figs. 2, 3, 4).

Replacing $\Psi$ by a homotopy equivalent map we may assume, without loss of generality, that

$$
\Psi\left(\mathbf{s}\left(b_{1} b_{2}\right)^{*}\right)=T+T_{21}, \quad \Psi\left(\mathbf{s}\left\{b_{1}, b_{2}\right\}^{*}\right)=\frac{1}{2}\left(T_{\cup}+T_{\cup o p p}\right) .
$$

So from now on we will assume that the map $\Psi$ (2.1) satisfies conditions (2.2).

Since the dg operad Braces acts on the Hochschild cochain complex $C^{\bullet}(\mathcal{A})$ of an $A_{\infty}$-algebra $\mathcal{A}$, the map $\Psi$ equips the Hochschild cochain complex $C^{\bullet}(\mathcal{A})$ with a structure of a Ger ${ }_{\infty}$-algebra. We will call it Tamarkin's Ger $_{\infty}$-structure and denote by

$$
C^{\bullet}(\mathcal{A})^{\Psi}
$$

the Hochschild cochain complex of $\mathcal{A}$ with the $\operatorname{Ger}_{\infty}$-structure coming from $\Psi$.

The choice of the homotopy class of $\Psi$ (2.1) (and hence the choice of Tamarkin's Ger $_{\infty}$-structure) is far from unique. In fact, it follows from [27, Theorem 1.2] that, the set of homotopy classes of maps (2.1) satisfying conditions (2.2) form a torsor for an infinite dimensional pro-algebraic group.

\footnotetext{
${ }^{6}$ Here, we use basis (1.8) in $\operatorname{Ger}^{\vee}(n)$.
} 
A simple degree bookkeeping in Braces shows that for every $n \geq 3$

$$
\Psi\left(\mathbf{s}\left(b_{1} b_{2} \cdots b_{n}\right)^{*}\right)=0 .
$$

Combining this observation with (2.2) we see that any Tamarkin's Ger $_{\infty}$-structure on $C^{\bullet}(\mathcal{A})$ satisfies the following remarkable property:

Property 2.1 The $\Lambda$ Lie $_{\infty}$ part of Tamarkin's $\operatorname{Ger}_{\infty}$-structure on $C^{\bullet}(\mathcal{A})$ coincides with the $\Lambda$ Lie-structure given by the Gerstenhaber bracket on $C^{\bullet}(\mathcal{A})$.

From now on, we only consider the case when $\mathcal{A}=A$, i.e. the free (graded) commutative algebra over $\mathbb{K}$ in variables $x^{1}, x^{2}, \ldots, x^{d}$ of (not necessarily zero) degrees $t_{1}, t_{2}, \ldots, t_{d}$, respectively. Furthermore, $V_{A}$ denotes the Gerstenhaber algebra of polyvector fields on the corresponding graded affine space, i.e.

$$
V_{A}:=S_{A}\left(\mathbf{s} \operatorname{Der}_{\mathbb{K}}(A)\right)
$$

It is known ${ }^{7}[16]$ that the canonical embedding

$$
V_{A} \hookrightarrow C^{\bullet}(A)
$$

is a quasi-isomorphism of cochain complexes, where $V_{A}$ is considered with the zero differential. In this paper, we refer to (2.4) as the Hochschild-Kostant-Rosenberg embedding.

Let us now consider the $\operatorname{Ger}_{\infty}$-algebra $C^{\bullet}(A)^{\Psi}$ for a chosen map $\Psi$ (2.1). By the first claim of Corollary 6.4 from Appendix B, there exists a Ger $_{\infty}$-quasi-isomorphism

$$
U_{\mathrm{Ger}}: V_{A} \rightsquigarrow C^{\bullet}(A)^{\Psi}
$$

whose linear term coincides with the Hochschild-Kostant-Rosenberg embedding.

Restricting $U_{\text {Ger }}$ to the $\Lambda^{2}$ coCom-coalgebra

$$
\Lambda^{2} \operatorname{coCom}\left(V_{A}\right)
$$

and taking into account Property 2.1 we get a $\Lambda$ Lie $_{\infty}$-quasi-isomorphism

$$
U_{\text {Lie }}: V_{A} \rightsquigarrow C^{\bullet}(A)
$$

of (dg) $\Lambda$ Lie-algebras.

Thus we deduced the main statement of Tamarkin's construction [24] which can be summarized as

\footnotetext{
7 Paper [16] treats only the case of usual (not graded) affine algebras. However, the proof of [16] can be generalized to the graded setting in a straightforward manner.
} 
Theorem 2.2 (Tamarkin [24]) Let A (resp. $V_{A}$ ) be the algebra of functions (resp. the algebra of polyvector fields) on a graded affine space. Let us consider the Hochschild cochain complex $C^{\bullet}(A)$ with the standard $\Lambda$ Lie-algebra structure. Then, for every map of dg operads $\Psi$ (2.1), there exists a $\Lambda \mathrm{Lie}_{\infty}$ quasi-isomorphism

$$
U_{\text {Lie }}: V_{A} \rightsquigarrow C^{\bullet}(A)
$$

which can be extended to a $\mathrm{Ger}_{\infty}$ quasi-isomorphism

$$
U_{\mathrm{Ger}}: V_{A} \rightsquigarrow C^{\bullet}(A)^{\Psi}
$$

where $V_{A}$ carries the standard Gerstenhaber algebra structure.

Remark 2.3 In this paper we tacitly assume that the linear part of every $\Lambda$ Lie $_{\infty}$ (resp. Ger ${ }_{\infty}$ ) quasi-isomorphism from $V_{A}$ to $C^{\bullet}(A)$ (resp. $C^{\bullet}(A)^{\Psi}$ ) coincides with the Hochschild-Kostant-Rosenberg embedding of polyvector fields into Hochschild cochains.

Since the above construction involves several choices it leaves the following two obvious questions:

Question A Is it possible to construct two homotopy inequivalent $\Lambda$ Lie $_{\infty}$-quasiisomorphisms (2.6) corresponding to the same map $\Psi$ (2.1)? And if no then

Question B Are $\Lambda$ Lie $_{\infty}$-quasi-isomorphisms $U_{\text {Lie }}$ and $\widetilde{U}_{\text {Lie }}(2.6)$ homotopy equivalent if so are the corresponding maps of dg operads $\Psi$ and $\widetilde{\Psi}(2.1)$ ?

The (expected) answer (NO) to Question A is given in the following proposition:

Proposition 2.4 Let $\Psi$ a map of dg operads (2.1) satisfying (2.2) and

$$
U_{\text {Lie }}, \widetilde{U}_{\text {Lie }}: V_{A} \rightsquigarrow C^{\bullet}(A)
$$

be $\Lambda$ Lie $_{\infty}$ quasi-morphisms which extend to Ger $_{\infty}$ quasi-isomorphisms

$$
U_{\mathrm{Ger}}, \widetilde{U}_{\mathrm{Ger}}: V_{A} \rightsquigarrow C^{\bullet}(A)^{\Psi}
$$

respectively. Then $U_{\mathrm{Lie}}$ is homotopy equivalent to $\widetilde{U}_{\mathrm{Lie}}$.

Proof This statement is essentially a consequence of general Corollary 6.4 from Appendix B.2.

Indeed, the second claim of Corollary 6.4 implies that Ger $_{\infty}$-morphisms (2.9) are homotopy equivalent. Hence so are their restrictions to the $\Lambda^{2}$ coCom-coalgebra

$$
\Lambda^{2} \operatorname{coCom}\left(V_{A}\right)
$$

which coincide with $U_{\text {Lie }}$ and $\widetilde{U}_{\text {Lie }}$, respectively. 
The expected answer (YES) to Question B is given in the following addition to Theorem 2.2:

Theorem 2.5 The homotopy type of $U_{\mathrm{Lie}}(2.6)$ depends only on the homotopy type of the map $\Psi$ (2.1).

Proof Let $\Psi$ and $\widetilde{\Psi}$ be maps of dg operads (2.1) satisfying (2.2) and let

$$
\begin{aligned}
& U_{\text {Lie }}: V_{A} \rightsquigarrow C^{\bullet}(A) \\
& \widetilde{U}_{\text {Lie }}: V_{A} \rightsquigarrow C^{\bullet}(A)
\end{aligned}
$$

be $\Lambda$ Lie $_{\infty}$ quasi-morphisms which extend to $\operatorname{Ger}_{\infty}$ quasi-isomorphisms

$$
U_{\mathrm{Ger}}: V_{A} \rightsquigarrow C^{\bullet}(A)^{\Psi} \text {, and } \widetilde{U}_{\mathrm{Ger}}: V_{A} \rightsquigarrow C^{\bullet}(A)^{\widetilde{\Psi}}
$$

respectively. Our goal is to show that if $\Psi$ is homotopy equivalent to $\widetilde{\Psi}$ then $U_{\text {Lie }}$ is homotopy equivalent to $\widetilde{U}_{\text {Lie }}$.

Let us denote by $\Omega^{\bullet}(\mathbb{K})$ the dg commutative algebra of polynomial forms on the affine line with the canonical coordinate $t$.

Since quasi-isomorphisms $\Psi, \widetilde{\Psi}: \operatorname{Ger}_{\infty} \rightarrow$ Braces are homotopy equivalent, we have $^{8}$ a map of dg operads

$$
\mathfrak{H}: \operatorname{Ger}_{\infty} \rightarrow \text { Braces } \otimes \Omega^{\bullet}(\mathbb{K})
$$

such that

$$
\Psi=p_{0} \circ \mathfrak{H}, \quad \text { and } \tilde{\Psi}=p_{1} \circ \mathfrak{H}
$$

where $p_{0}$ and $p_{1}$ are the canonical maps (of dg operads)

$$
\begin{aligned}
& p_{0}, p_{1}: \text { Braces } \otimes \Omega^{\bullet}(\mathbb{K}) \rightarrow \text { Braces, } \\
& p_{0}(v):=\left.v\right|_{d t=0, t=0, \quad p_{1}(v):=\left.v\right|_{d t=0, t=1} .}
\end{aligned}
$$

The map $\mathfrak{H}$ induces a $\operatorname{Ger}_{\infty}$-structure on $C^{\bullet}(A) \otimes \Omega^{\bullet}(\mathbb{K})$ such that the evaluation maps (which we denote by the same letters)

$$
\begin{aligned}
& p_{0}: C^{\bullet}(A) \otimes \Omega^{\bullet}(\mathbb{K}) \rightarrow C^{\bullet}(A)^{\Psi}, \quad p_{0}(v):=\left.v\right|_{d t=0, t=0}, \\
& p_{1}: C^{\bullet}(A) \otimes \Omega^{\bullet}(\mathbb{K}) \rightarrow C^{\bullet}(A)^{\widetilde{\Psi}}, \quad p_{1}(v):=\left.v\right|_{d t=0, t=1}
\end{aligned}
$$

are strict quasi-isomorphisms of the corresponding $\mathrm{Ger}_{\infty}$-algebras.

So, in this proof, we consider the cochain complex $C^{\bullet}(A) \otimes \Omega^{\bullet}(\mathbb{K})$ with the $\operatorname{Ger}_{\infty}$ structure coming from $\mathfrak{H}$ (2.13). The same degree bookkeeping argument in Braces shows that ${ }^{9}$

\footnotetext{
${ }^{8}$ For justification of this step see, for example, [5, Section 5.1].

${ }^{9}$ Here, we use basis (1.8) in $\operatorname{Ger}^{\vee}(n)$.
} 


$$
\mathfrak{H}\left(\mathbf{s}\left(b_{1} b_{2} \cdots b_{n}\right)^{*}\right)=0 .
$$

Hence, the $\Lambda$ Lie $_{\infty}$ part of the $\operatorname{Ger}_{\infty}$-structure on $C^{\bullet}(A) \otimes \Omega^{\bullet}(\mathbb{K})$ coincides with the $\Lambda$ Lie-structure given by the Gerstenhaber bracket extended from $C^{\bullet}(A)$ to $C^{\bullet}(A) \otimes$ $\Omega^{\bullet}(\mathbb{K})$ to by $\Omega^{\bullet}(\mathbb{K})$-linearity.

Since the canonical embedding

$$
P \mapsto P \otimes 1: C^{\bullet}(A) \hookrightarrow C^{\bullet}(A) \otimes \Omega^{\bullet}(\mathbb{K})
$$

is a quasi-isomorphism of cochain complexes, Corollary 6.4 from Appendix B.2 implies that there exists a $\mathrm{Ger}_{\infty}$ quasi-isomorphism

$$
U_{\mathrm{Ger}}^{\mathfrak{H}}: V_{A} \rightsquigarrow C^{\bullet}(A) \otimes \Omega^{\bullet}(\mathbb{K}),
$$

where $V_{A}$ is considered with the standard Gerstenhaber structure.

Since the $\Lambda \mathrm{Lie}_{\infty}$ part of the $\operatorname{Ger}_{\infty}$-structure on $C^{\bullet}(A) \otimes \Omega^{\bullet}(\mathbb{K})$ coincides with the standard $\Lambda$ Lie-structure, the restriction of $U_{\mathrm{Ger}}^{\mathfrak{H}}$ to the $\Lambda^{2}$ coCom-coalgebra $\Lambda^{2} \operatorname{coCom}\left(V_{A}\right)$ gives us a homotopy connecting the $\Lambda \mathrm{Lie}_{\infty}$ quasi-isomorphism

$$
\left.p_{0} \circ U_{\mathrm{Ger}}^{\mathfrak{H}}\right|_{\Lambda^{2} \operatorname{coCom}\left(V_{A}\right)}: V_{A} \rightsquigarrow C^{\bullet}(A)
$$

to the $\Lambda \mathrm{Lie}_{\infty}$ quasi-isomorphism

$$
\left.p_{1} \circ U_{\mathrm{Ger}}^{\mathfrak{H}}\right|_{\Lambda^{2} \operatorname{coCom}\left(V_{A}\right)}: V_{A} \rightsquigarrow C^{\bullet}(A),
$$

where $p_{0}$ and $p_{1}$ are evaluation maps (2.14).

Let us now observe that $\Lambda$ Lie $_{\infty}$ quasi-isomorphisms (2.17) and (2.18) extend to $\mathrm{Ger}_{\infty}$ quasi-isomorphisms

$$
p_{0} \circ U_{\mathrm{Ger}}^{\mathfrak{H}}: V_{A} \rightsquigarrow C^{\bullet}(A)^{\Psi}, \quad \text { and } \quad p_{1} \circ U_{\mathrm{Ger}}^{\mathfrak{H}}: V_{A} \rightsquigarrow C^{\bullet}(A)^{\widetilde{\Psi}}
$$

respectively. Hence, by Proposition $2.4, \Lambda$ Lie $_{\infty}$ quasi-isomorphism (2.17) is homotopy equivalent to (2.10) and $\Lambda \mathrm{Lie}_{\infty}$ quasi-isomorphism (2.18) is homotopy equivalent to $(2.11)$. lent.

Thus $\Lambda$ Lie $_{\infty}$ quasi-isomorphisms (2.10) and (2.11) are indeed homotopy equiva-

The general conclusion of this section is that Tamarkin's construction $[15,24]$ gives us a map

$$
\mathfrak{T}: \pi_{0}\left(\operatorname{Ger}_{\infty} \rightarrow \text { Braces }\right) \rightarrow \pi_{0}\left(V_{A} \rightsquigarrow C^{\bullet}(A)\right)
$$

from the set $\pi_{0}$ (Ger $\infty \rightarrow$ Braces) of homotopy classes of operad morphisms (2.1) satisfying conditions (2.2) to the set $\pi_{0}\left(V_{A} \rightsquigarrow C^{\bullet}(A)\right)$ of homotopy classes of $\Lambda \mathrm{Lie}_{\infty}$-morphisms from $V_{A}$ to $C^{\bullet}(A)$ whose linear term is the Hochschild-KostantRosenberg embedding. 


\section{Actions of GRT 1}

Let $\mathcal{C}$ be a coaugmented cooperad in the category of graded vector spaces and $\mathcal{C}_{\circ}$ be the cokernel of the coaugmentation. We assume that $\mathcal{C}(0)=\mathbf{0}$ and $\mathcal{C}(1)=\mathbb{K}$.

Let us denote by

$$
\operatorname{Der}^{\prime}(\operatorname{Cobar}(\mathcal{C}))
$$

the dg Lie algebra of derivation $\mathcal{D}$ of $\operatorname{Cobar}(\mathcal{C})$ satisfying the condition

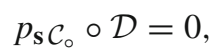

where $p_{\mathbf{s}} \mathcal{C}_{\circ}$ is the canonical projection $\operatorname{Cobar}(\mathcal{C}) \rightarrow \mathbf{s} \mathcal{C}_{\circ}$. Conditions $\mathcal{C}(0)=\mathbf{0}, \mathcal{C}(1)=$ $\mathbb{K}$ and (3.2) imply that $\operatorname{Der}^{\prime}(\operatorname{Cobar}(\mathcal{C}))^{0}$ and $H^{0}\left(\operatorname{Der}^{\prime}(\operatorname{Cobar}(\mathcal{C}))\right)$ are pronilpotent Lie algebras.

In this paper, we are mostly interested in the case when $\mathcal{C}=\Lambda^{2}$ coCom and $\mathcal{C}=\operatorname{Ger}^{\vee}$. The corresponding dg operads $\Lambda$ Lie $_{\infty}=\operatorname{Cobar}\left(\Lambda^{2} \operatorname{coCom}\right)$ and $\operatorname{Ger}_{\infty}=$ $\operatorname{Cobar}\left(\right.$ Ger $\left.^{\vee}\right)$ govern $\Lambda$ Lie $_{\infty}$ and Ger $_{\infty}$ algebras, respectively.

A simple degree bookkeeping shows that

$$
\operatorname{Der}^{\prime}\left(\Lambda \mathrm{Lie}_{\infty}\right)^{\leq 0}=\mathbf{0}
$$

i.e. the dg Lie algebra $\operatorname{Der}^{\prime}\left(\Lambda\right.$ Lie $\left._{\infty}\right)$ does not have non-zero elements in degrees $\leq 0$. In particular, the Lie algebra $H^{0}\left(\operatorname{Der}^{\prime}\left(\Lambda \mathrm{Lie}_{\infty}\right)\right)$ is zero.

On the other hand, the Lie algebra

$$
\mathfrak{g}=H^{0}\left(\operatorname{Der}^{\prime}\left(\operatorname{Ger}_{\infty}\right)\right)
$$

is much more interesting. According to Willwacher's theorem [27, Theorem 1.2], this Lie algebra is isomorphic to the pro-nilpotent part $\mathfrak{g r t}_{1}$ of the GrothendieckTeichmueller Lie algebra $\mathfrak{g r t}[1, \operatorname{Section} 4.2]$. Hence, the group $\exp (\mathfrak{g})$ is isomorphic to the group $\mathrm{GRT} T_{1}=\exp \left(\mathfrak{g r t}_{1}\right)$.

Let us now describe how the group $\exp (\mathfrak{g}) \cong \mathrm{GRT}_{1}$ acts both on the source and the target of Tamarkin's map $\mathfrak{T}$ (2.20).

\subsection{The action of GRT 1 on $\pi_{0}\left(\mathrm{Ger}_{\infty} \rightarrow\right.$ Braces)}

Let $v$ be a vector of $\mathfrak{g}$ represented by a (degree zero) cocycle $\mathcal{D} \in \operatorname{Der}^{\prime}\left(\operatorname{Ger}_{\infty}\right)$. Since the Lie algebra $\operatorname{Der}^{\prime}\left(\operatorname{Ger}_{\infty}\right)^{0}$ is pro-nilpotent, $\mathcal{D}$ gives us an automorphism

$$
\exp (\mathcal{D})
$$

of the operad $\operatorname{Ger}_{\infty}$. 
Let $\Psi$ be a quasi-isomorphism of dg operads (2.1). Due to Proposition B.2 in [22], the homotopy type of the composition

$$
\Psi \circ \exp (\mathcal{D})
$$

does not depend on the choice of the cocycle $\mathcal{D}$ in the cohomology class $v$. Furthermore, for every pair of (degree zero) cocycles $\mathcal{D}, \widetilde{\mathcal{D}} \in \operatorname{Der}^{\prime}\left(\operatorname{Ger}_{\infty}\right)$ we have

$$
\Psi \circ \exp (\mathcal{D}) \circ \exp (\widetilde{\mathcal{D}})=\Psi \circ \exp (\mathrm{CH}(\mathcal{D}, \widetilde{\mathcal{D}}))
$$

where $\mathrm{CH}(x, y)$ denotes the Campbell-Hausdorff series in symbols $x, y$.

Thus the assignment

$$
\Psi \rightarrow \Psi \circ \exp (\mathcal{D})
$$

induces a right action of the group $\exp (\mathfrak{g})$ on the set $\pi_{0}\left(\mathrm{Ger}_{\infty} \rightarrow\right.$ Braces) of homotopy classes of operad morphisms (2.1).

\subsection{The action of $\mathrm{GRT}_{1}$ on $\pi_{0}\left(V_{A} \rightsquigarrow C^{\bullet}(A)\right)$}

Let us now show that $\exp (\mathfrak{g}) \cong \mathrm{GRT}_{1}$ also acts on the set $\pi_{0}\left(V_{A} \rightsquigarrow C^{\bullet}(A)\right)$ of homotopy classes of $\Lambda$ Lie $_{\infty}$-morphisms from $V_{A}$ to $C^{\bullet}(A)$.

For this purpose, we denote by

$$
\text { Act }_{\text {stan }}: \operatorname{Ger}_{\infty} \rightarrow \text { End }_{V_{A}}
$$

the operad map corresponding to the standard Gerstenhaber structure on $V_{A}$.

Then, given a cocycle $\mathcal{D} \in \operatorname{Der}^{\prime}\left(\operatorname{Ger}_{\infty}\right)$ representing $v \in \mathfrak{g}$, we may precompose map (3.6) with automorphism (3.5). This way, we equip the graded vector space $V_{A}$ with a new $\operatorname{Ger}_{\infty}$-structure $Q^{\exp (\mathcal{D})}$ whose binary operations are the standard ones. Therefore, by Corollary 6.3 from Appendix B.1, there exists a Ger $\infty$ quasiisomorphism

$$
U_{\text {corr }}: V_{A} \rightarrow V_{A}^{Q^{\exp (\mathcal{D})}}
$$

from $V_{A}$ with the standard Gerstenhaber structure to $V_{A}$ with the $\operatorname{Ger}_{\infty}$-structure $Q^{\exp (\mathcal{D})}$.

Due to observation (3.3), the restriction of $\mathcal{D}$ onto the suboperad Cobar $\left(\Lambda^{2} \mathrm{coCom}\right)$ $\subset \operatorname{Cobar}\left(\operatorname{Ger}^{\vee}\right)$ is zero. Hence, for every degree zero cocycle $\mathcal{D} \in \operatorname{Der}^{\prime}\left(\operatorname{Ger}_{\infty}\right)$, we have

$$
\left.\exp (\mathcal{D})\right|_{\operatorname{Cobar}\left(\Lambda^{2} \operatorname{coCom}\right)}=\operatorname{Id}: \operatorname{Cobar}\left(\Lambda^{2} \operatorname{coCom}\right) \rightarrow \operatorname{Cobar}\left(\Lambda^{2} \operatorname{coCom}\right) .
$$

Therefore the $\Lambda \mathrm{Lie}_{\infty}$-part of the $\operatorname{Ger}_{\infty}$-structure $Q^{\exp (\mathcal{D})}$ coincides with the standard $\Lambda$ Lie-structure on $V_{A}$ given by the Schouten bracket. Hence the restriction of the 
$\operatorname{Ger}_{\infty}$ quasi-isomorphism $U_{\text {corr }}$ onto the $\Lambda^{2} \operatorname{coCom}$-coalgebra $\Lambda^{2} \operatorname{coCom}\left(V_{A}\right)$ gives us a $\Lambda$ Lie $_{\infty}$-automorphism

$$
U^{\mathcal{D}}: V_{A} \rightsquigarrow V_{A} \text {. }
$$

Note that, for a fixed $\operatorname{Ger}_{\infty}$-structure $Q^{\exp (\mathcal{D})}, \operatorname{Ger}_{\infty}$ quasi-isomorphism (3.7) is far from unique. However, the second statement of Corollary 6.4 implies that the homotopy class of (3.7) is unique. Therefore, the assignment

$$
\mathcal{D} \mapsto\left[U^{\mathcal{D}}\right]
$$

is a well defined map from the set of degree zero cocycles of $\operatorname{Der}^{\prime}\left(\operatorname{Ger}_{\infty}\right)$ to homotopy classes of $\Lambda$ Lie $\infty_{\infty}$-automorphisms of $V_{A}$.

This statement can be strengthened further:

Proposition 3.1 The homotopy type of $U^{\mathcal{D}}$ does not depend on the choice of the representative $\mathcal{D}$ of the cohomology class $v$. Furthermore, for any pair of degree zero cocycles $\mathcal{D}_{1}, \mathcal{D}_{2} \in \operatorname{Der}^{\prime}\left(\operatorname{Ger}_{\infty}\right)$, the composition $U^{\mathcal{D}_{1}} \circ U^{\mathcal{D}_{2}}$ is homotopy equivalent to $U^{\mathrm{CH}\left(\mathcal{D}_{1}, \mathcal{D}_{2}\right)}$, where $\mathrm{CH}(x, y)$ denotes the Campbell-Hausdorff series in symbols $x, y$.

Let us postpone the technical Proof of Proposition 3.1 to Sect. 3.4 and observe that this proposition implies the following statement:

Corollary 3.2 Let $\mathcal{D}$ be a degree zero cocycle in $\operatorname{Der}^{\prime}\left(\mathrm{Ger}_{\infty}\right)$ representing a cohomology class $v \in \mathfrak{g}$ and let $U_{\mathrm{Lie}}$ be a $\Lambda \mathrm{Lie}_{\infty}$ quasi-isomorphism from $V_{A}$ to $C^{\bullet}(A)$. The assignment

$$
U_{\text {Lie }} \mapsto U_{\text {Lie }} \circ U^{\mathcal{D}}
$$

induces a right action of the group $\exp (\mathfrak{g})$ on the set $\pi_{0}\left(V_{A} \rightsquigarrow C^{\bullet}(A)\right)$ of homotopy classes of $\Lambda$ Lie $_{\infty}$-morphisms from $V_{A}$ to $C^{\bullet}(A)$.

From now on, by abuse of notation, we denote by $U^{\mathcal{D}}$ any representative in the homotopy class of $\Lambda$ Lie $_{\infty}$-automorphism (3.9).

\subsection{The theorem on $\mathrm{GRT}_{1}$-equivariance}

The following theorem is the main result of this paper:

Theorem 3.3 Let $\pi_{0}$ (Ger G $_{\infty} \rightarrow$ Braces) be the set of homotopy classes of operad maps (2.1) from the dg operad $\mathrm{Ger}_{\infty}$ governing homotopy Gerstenhaber algebras to the dg operad Braces of brace trees. Let $\pi_{0}\left(V_{A} \rightsquigarrow C^{\bullet}(A)\right)$ be the set of homotopy classes of $\Lambda$ Lie $_{\infty}$ quasi-isomorphisms ${ }^{10}$ from the algebra $V_{A}$ of polyvector fields to

\footnotetext{
10 We tacitly assume that operad maps (2.1) satisfies conditions (2.2) and $\Lambda$ Lie $_{\infty}$ quasi-isomorphisms $V_{A} \rightsquigarrow C^{\bullet}(A)$ extend the Hochschild-Kostant-Rosenberg embedding.
} 
the algebra $C^{\bullet}(A)$ of Hochschild cochains of a graded affine space. Then Tamarkin's map $\mathfrak{T}(2.20)$ commutes with the action of the group $\exp (\mathfrak{g})$ which corresponds to Lie algebra (3.4).

Proof Following [22, Section 3], [13], we will denote by Cyl(Ger $\left.{ }^{\vee}\right)$ the 2-colored dg operad whose algebras are pairs $(V, W)$ with the data

1. a $\operatorname{Ger}_{\infty}$-structure on $V$,

2. a $\operatorname{Ger}_{\infty}$-structure on $W$, and

3. a $\operatorname{Ger}_{\infty}$-morphism $F$ from $V$ to $W$, i.e. a homomorphism of corresponding dg $\operatorname{Ger}^{\vee}$-coalgebras $\operatorname{Ger}^{\vee}(V) \rightarrow \operatorname{Ger}^{\vee}(W)$.

In fact, if we forget about the differential, then the operad $\mathrm{Cyl}\left(\mathrm{Ger}^{\vee}\right)$ is a free operad on a certain 2-colored collection $\mathcal{M}\left(\mathrm{Ger}^{\vee}\right)$ naturally associated to $\mathrm{Ger}^{\vee}$.

Let us denote by

$$
\operatorname{Der}^{\prime}\left(\operatorname{Cyl}\left(\operatorname{Ger}^{\vee}\right)\right)
$$

the dg Lie algebra of derivations $\mathcal{D}$ of $\operatorname{Cyl}\left(\mathrm{Ger}^{\vee}\right)$ subject to the condition ${ }^{11}$

$$
p \circ \mathcal{D}=0
$$

where $p$ is the canonical projection from $\mathrm{Cyl}\left(\mathrm{Ger}^{\vee}\right)$ onto $\mathcal{M}\left(\mathrm{Ger}^{\vee}\right)$.

The restrictions to the first color part and the second color part of $\mathrm{Cyl}\left(\mathrm{Ger}^{\vee}\right)$, respectively, give us natural maps of dg Lie algebras

$$
\operatorname{res}_{1}, \operatorname{res}_{2}: \operatorname{Der}^{\prime}\left(\operatorname{Cyl}\left(\operatorname{Ger}^{\vee}\right)\right) \rightarrow \operatorname{Der}^{\prime}\left(\operatorname{Ger}_{\infty}\right)
$$

and, due to [22, Theorem 4.3], res 1 and res $_{2}$ are chain homotopic quasi-isomorphisms.

Therefore, for every $v \in \mathfrak{g}$ there exists a degree zero cocycle

$$
\mathcal{D} \in \operatorname{Der}^{\prime}\left(\operatorname{Cyl}\left(\operatorname{Ger}^{\vee}\right)\right)
$$

such that both $\operatorname{res}_{1}(\mathcal{D})$ and $\operatorname{res}_{2}(\mathcal{D})$ represent the cohomology class $v$.

Let

$$
U_{\mathrm{Ger}}: V_{A} \rightsquigarrow C^{\bullet}(A)^{\Psi}
$$

be a $\operatorname{Ger}_{\infty}$-morphism from $V_{A}$ to $C^{\bullet}(A)$ which restricts to a $\Lambda$ Lie $\infty_{\infty}$-morphism

$$
U_{\text {Lie }}: V_{A} \rightarrow C^{\bullet}(A)
$$

The triple consisting of

- the standard Gerstenhaber structure on $V_{A}$,

\footnotetext{
11 It is condition (3.12) which guarantees that any degree zero cocycle in $\operatorname{Der}^{\prime}\left(\mathrm{Cyl}\left(\mathrm{Ger}^{\vee}\right)\right)$ can be exponentiated to an automorphism of $\mathrm{Cyl}\left(\mathrm{Ger}^{\vee}\right)$.
} 
- the $\operatorname{Ger}_{\infty}$-structure on $C^{\bullet}(A)$ coming from a map $\Psi$, and

- $\operatorname{Ger}_{\infty}$-morphism (3.15)

gives us a map of dg operads

$$
U_{\mathrm{Cyl}}: \operatorname{Cyl}\left(\mathrm{Ger}^{\vee}\right) \rightarrow \mathrm{End}_{V_{A}, C^{\bullet}(A)}
$$

from $\mathrm{Cyl}\left(\mathrm{Ger}^{\vee}\right)$ to the 2-colored endomorphism operad $\mathrm{End}_{V_{A}, C^{\bullet}(A)}$ of the pair $\left(V_{A}, C^{\bullet}(A)\right)$.

Precomposing $U_{\mathrm{Cyl}}$ with the endomorphism

$$
\exp (\mathcal{D}): \operatorname{Cyl}\left(\mathrm{Ger}^{\vee}\right) \rightarrow \operatorname{Cyl}\left(\mathrm{Ger}^{\vee}\right)
$$

we get another operad map

$$
U_{\mathrm{Cyl}} \circ \exp (\mathcal{D}): \operatorname{Cyl}\left(\mathrm{Ger}^{\vee}\right) \rightarrow \operatorname{End}_{V_{A}, C^{\bullet}(A)}
$$

which corresponds to the triple consisting of

- the new $\operatorname{Ger}_{\infty}$-structure $Q^{\exp \left(\operatorname{res}_{1}(\mathcal{D})\right)}$ on $V_{A}$,

- the $\operatorname{Ger}_{\infty}$-structure on $C^{\bullet}(A)$ corresponding to $\Psi \circ \exp \left(\operatorname{res}_{2}(\mathcal{D})\right)$, and

- a $\operatorname{Gr}_{\infty}$ quasi-isomorphism

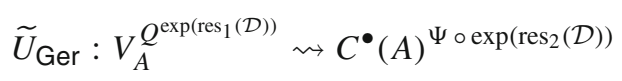

Due to technical Proposition 7.1 proved in Appendix $\mathrm{C}$ below, the restriction of the Ger $\infty$ quasi-isomorphism $\widetilde{U}_{\mathrm{Ger}}(3.19)$ to $\Lambda^{2} \operatorname{coCom}\left(V_{A}\right)$ gives us the same $\Lambda \mathrm{Lie}_{\infty^{-}}$ morphism (3.16).

On the other hand, by Corollary 6.3 from Appendix B.1, there exists a Ger $_{\infty}$ quasi-isomorphism

$$
U_{\text {corr }}: V_{A} \rightarrow V_{A}^{Q^{\exp \left(\operatorname{res}_{1}(\mathcal{D})\right)}}
$$

from $V_{A}$ with the standard Gerstenhaber structure to $V_{A}$ with the new $\operatorname{Ger}_{\infty}$-structure $Q^{\exp \left(\operatorname{res}_{1}(\mathcal{D})\right)}$.

Thus, composing $U_{\text {corr }}$ with $\widetilde{U}_{\text {Ger }}$ (3.19), we get a Ger $_{\infty}$ quasi-isomorphism

$$
U_{\mathrm{Ger}}^{\exp (\mathcal{D})}: V_{A} \rightsquigarrow C^{\bullet}(A)^{\Psi \circ \exp \left(\operatorname{res}_{2}(\mathcal{D})\right)}
$$

from $V_{A}$ with the standard Gerstenhaber structure to $C^{\bullet}(A)$ with the $\operatorname{Ger}_{\infty}$-structure coming from $\Psi \circ \exp \left(\operatorname{res}_{2}(\mathcal{D})\right)$.

The restriction of this $\operatorname{Ger}_{\infty}$-morphism $U_{\mathrm{Ger}}^{\exp (\mathcal{D})}$ to $\Lambda^{2} \operatorname{coCom}\left(V_{A}\right)$ gives us the $\Lambda$ Lie $_{\infty}$-morphism

$$
U_{\text {Lie }} \circ U^{\mathrm{res}_{1}(\mathcal{D})}
$$


where $U^{\text {res }_{1}(\mathcal{D})}$ is the $\Lambda$ Lie ${ }_{\infty}$-automorphism of $V_{A}$ obtained by restricting (3.20) to $\Lambda^{2} \operatorname{coCom}\left(V_{A}\right)$.

Since both cocycles $\operatorname{res}_{1}(\mathcal{D})$ and $\operatorname{res}_{2}(\mathcal{D})$ of $\operatorname{Der}^{\prime}\left(\operatorname{Ger}_{\infty}\right)$ represent the same cohomology class $v \in \mathfrak{g}$, Theorem 3.3 follows.

\subsection{The proof of Proposition 3.1}

Let $\mathcal{D}$ and $\widetilde{\mathcal{D}}$ be two cohomologous cocycles in $\operatorname{Der}^{\prime}\left(\operatorname{Ger}_{\infty}\right)$ and let $Q^{\exp (\mathcal{D})}, Q^{\exp (\widetilde{\mathcal{D}})}$ be $\operatorname{Ger}_{\infty}$-structures on $V_{A}$ corresponding to the operad maps

$$
\begin{aligned}
& \operatorname{Act}_{\text {stan }} \circ \exp (\mathcal{D}): \operatorname{Ger}_{\infty} \rightarrow \mathrm{End}_{V_{A}}, \\
& \operatorname{Act}_{\text {stan }} \circ \exp (\widetilde{\mathcal{D}}): \operatorname{Ger}_{\infty} \rightarrow \mathrm{End}_{V_{A}},
\end{aligned}
$$

respectively. Here $\mathrm{Act}_{s t a n}$ is the map $\mathrm{Ger}_{\infty} \rightarrow \mathrm{End}_{V_{A}}$ corresponding to the standard Gerstenhaber structure on $V_{A}$.

Due to Proposition B.2 in [22], operad maps (3.23) and (3.24) are homotopy equivalent. Hence there exists a $\operatorname{Ger}_{\infty}$-structure $Q_{t}$ on $V_{A} \otimes \Omega^{\bullet}(\mathbb{K})$ such that the evaluation maps

$$
\begin{array}{ll}
p_{0}: V_{A} \otimes \Omega^{\bullet}(\mathbb{K}) \rightarrow V_{A}^{Q^{\exp (\mathcal{D})},} & p_{0}(v):=\left.v\right|_{d t=0, t=0}, \\
p_{1}: V_{A} \otimes \Omega^{\bullet}(\mathbb{K}) \rightarrow V_{A}^{Q^{\exp (\tilde{\mathcal{D}})},}, \quad p_{1}(v):=\left.v\right|_{d t=0, t=1}
\end{array}
$$

are strict quasi-isomorphisms of the corresponding Ger $_{\infty}$-algebras.

Furthermore, observation (3.3) implies that the restriction of a homotopy connecting the automorphisms $\exp (\mathcal{D})$ and $\exp (\widetilde{\mathcal{D}})$ of $\operatorname{Ger}_{\infty}$ to the suboperad $\Lambda$ Lie $\operatorname{soincides}_{\infty}$ with the identity map on $\Lambda$ Lie $_{\infty}$ for every $t$. Therefore, the $\Lambda$ Lie $_{\infty}$-part of the Ger $_{\infty}$ structure $Q_{t}$ on $V_{A} \otimes \Omega^{\bullet}(\mathbb{K})$ coincides with the standard $\Lambda$ Lie-structure given by the Schouten bracket.

Since tensoring with $\Omega^{\bullet}(\mathbb{K})$ does not change cohomology, Corollary 6.4 from Appendix B.2 implies that the canonical embedding $V_{A} \hookrightarrow V_{A} \otimes \Omega^{\bullet}(\mathbb{K})$ can be extended to a $\mathrm{Ger}_{\infty}$ quasi-isomorphism

$$
U_{\text {corr }}^{\mathfrak{H}}: V_{A} \rightsquigarrow V_{A} \otimes \Omega^{\bullet}(\mathbb{K})
$$

from $V_{A}$ with the standard Gerstenhaber structure to $V_{A} \otimes \Omega^{\bullet}(\mathbb{K})$ with the $\operatorname{Ger}_{\infty^{-}}$ structure $Q_{t}$.

Since the $\Lambda$ Lie $_{\infty}$-part of the $\operatorname{Ger}_{\infty}$-structure $Q_{t}$ on $V_{A} \otimes \Omega^{\bullet}(\mathbb{K})$ coincides with the standard $\Lambda$ Lie-structure given by the Schouten bracket, the restriction of $U_{\text {corr }}^{\mathfrak{H}}$ onto $\Lambda^{2} \operatorname{coCom}\left(V_{A}\right)$ gives us a homotopy connecting the $\Lambda$ Lie $_{\infty}$-automorphisms

$$
\left.p_{0} \circ U_{\operatorname{corr}}^{\mathfrak{H}}\right|_{\Lambda^{2} \operatorname{coCom}\left(V_{A}\right)}: V_{A} \rightsquigarrow V_{A}
$$

and

$$
\left.p_{1} \circ U_{\text {corr }}^{\mathfrak{H}}\right|_{\Lambda^{2} \operatorname{coCom}\left(V_{A}\right)}: V_{A} \rightsquigarrow V_{A}
$$


Due to the second part of Corollary $6.4, \Lambda \mathrm{Lie}_{\infty}$-automorphism (3.27) is homotopy

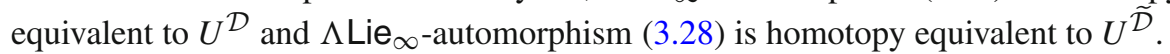

Thus the homotopy type of $U^{\mathcal{D}}$ is indeed independent of the representative $\mathcal{D}$ of the cohomology class.

To prove the second claim of Proposition 3.1, we will need to use the 2-colored $\mathrm{dg}$ operad Cyl $\left(\mathrm{Ger}^{\vee}\right)$ recalled in the Proof of Theorem 3.3 above. Moreover, we need [22, Theorem 4.3] which implies that restrictions (3.13) are homotopic quasi-isomorphisms of cochain complexes.

Let $\mathcal{D}_{1}$ and $\mathcal{D}_{2}$ be degree zero cocycles in $\operatorname{Der}^{\prime}\left(\operatorname{Ger}_{\infty}\right)$ and let $Q^{\exp \left(\mathcal{D}_{1}\right)}$ be the $\operatorname{Ger}_{\infty}$-structure on $V_{A}$ which comes from the composition

$$
\operatorname{Act}_{\text {stan }} \circ \exp \left(\mathcal{D}_{1}\right): \operatorname{Ger}_{\infty} \rightarrow \text { End }_{V_{A}}
$$

where Act stan $_{\text {denotes the map }} \mathrm{Ger}_{\infty} \rightarrow \mathrm{End}_{V_{A}}$ corresponding to the standard Gerstenhaber structure on $V_{A}$.

Let $U_{\mathrm{Ger}, 1}$ be a $\operatorname{Ger}_{\infty}$-quasi-isomorphism

$$
U_{\mathrm{Ger}, 1}: V_{A} \rightsquigarrow V_{A}^{Q^{\exp \left(\mathcal{D}_{1}\right)},}
$$

where the source is considered with the standard Gerstenhaber structure.

By construction, the $\Lambda \mathrm{Lie}_{\infty}$-automorphism

$$
U^{\mathcal{D}_{1}}: V_{A} \rightsquigarrow V_{A}
$$

is the restriction of $U_{\mathrm{Ger}, 1}$ onto $\Lambda^{2} \operatorname{coCom}\left(V_{A}\right)$.

Let us denote by $U_{\mathrm{Cyl}}^{V_{A}}$ the operad map

$$
U_{\mathrm{Cyl}}^{V_{A}}: \operatorname{Cyl}\left(\mathrm{Ger}^{\vee}\right) \rightarrow \mathrm{End}_{V_{A}, V_{A}}
$$

which corresponds to the triple:

- the standard Gerstenhaber structure on the first copy of $V_{A}$,

- the $\operatorname{Ger}_{\infty}$-structure $Q^{\exp \left(\mathcal{D}_{1}\right)}$ on the second copy of $V_{A}$, and

- the chosen Ger $_{\infty}$ quasi-isomorphism in (3.30).

Due to [22, Theorem 4.3], there exists a degree zero cocycle $\mathcal{D}_{\mathrm{Cyl}}$ in $\mathrm{Der}^{\prime}$ $\left(\mathrm{Cyl}\left(\mathrm{Ger}^{\vee}\right)\right)$ for which the cocycles

$$
\mathcal{D}:=\operatorname{res}_{1}\left(\mathcal{D}_{\mathrm{Cyl}}\right), \quad \mathcal{D}^{\prime}:=\operatorname{res}_{2}\left(\mathcal{D}_{\mathrm{Cyl}}\right)
$$

are both cohomologous to the given cocycle $\mathcal{D}_{2}$.

Precomposing the map $U_{\mathrm{Cyl}}^{V_{A}}$ with the automorphism $\exp \left(\mathcal{D}_{\mathrm{Cyl}}\right)$ we get a new $\operatorname{Cyl}\left(\mathrm{Ger}^{\vee}\right)$-algebra structure on the pair $\left(V_{A}, V_{A}\right)$ which corresponds to the triple

- the $\operatorname{Ger}_{\infty}$-structure $Q^{\exp (\mathcal{D})}$ on the first copy of $V_{A}$,

- the $\operatorname{Ger}_{\infty}$-structure $Q^{\exp \left(\operatorname{CH}\left(\mathcal{D}_{1}, \mathcal{D}^{\prime}\right)\right)}$ on the second copy of $V_{A}$, and 
- a $\operatorname{Gr}_{\infty}$ quasi-isomorphism

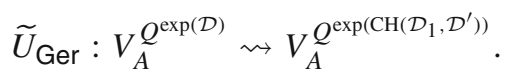

Let us observe that, due to Proposition 7.1 from Appendix C, the restriction of $\widetilde{U}_{\text {Ger }}$ onto $\Lambda^{2} \operatorname{coCom}\left(V_{A}\right)$ coincides with the restriction of (3.30) onto $\Lambda^{2} \operatorname{coCom}\left(V_{A}\right)$. Hence,

$$
\left.\widetilde{U}_{\mathrm{Ger}}\right|_{\Lambda^{2} \operatorname{coCom}\left(V_{A}\right)}=U^{\mathcal{D}_{1}}
$$

where $U^{\mathcal{D}_{1}}$ is a $\Lambda$ Lie $_{\infty}$-automorphism of $V_{A}$ corresponding ${ }^{12}$ to $\mathcal{D}_{1}$.

Recall that there exists a Ger $_{\infty}$ quasi-isomorphism

$$
U_{\mathrm{Ger}}: V_{A} \rightsquigarrow V_{A}^{Q^{\exp (\mathcal{D})}} \text {. }
$$

where the source is considered with the standard Gerstenhaber structure. Furthermore, since $\mathcal{D}$ is cohomologous to $\mathcal{D}_{2}$, the first claim of Proposition 3.1 implies that the

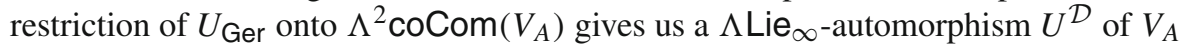
which is homotopy equivalent to $U^{\mathcal{D}_{2}}$.

Let us also observe that the composition $\widetilde{U}_{\text {Ger }} \circ U_{\text {Ger gives us a }}$ Ger $\infty$ quasiisomorphism

$$
\widetilde{U}_{\mathrm{Ger}} \circ U_{\mathrm{Ger}}: V_{A} \rightsquigarrow V_{A}^{Q^{\exp \left(\mathrm{CH}\left(\mathcal{D}_{1}, \mathcal{D}^{\prime}\right)\right)}}
$$

Hence, the restriction of $\widetilde{U}_{\text {Ger }} \circ U_{\text {Ger }}$ gives us a $\Lambda$ Lie $\infty_{\infty}$-automorphism of $V_{A}$ corresponding to $\mathrm{CH}\left(\mathcal{D}_{1}, \mathcal{D}^{\prime}\right)$. Due to (3.33), this $\Lambda$ Lie $_{\infty}$-automorphism coincides with

$$
U^{\mathcal{D}_{1}} \circ U^{\mathcal{D}}
$$

Since $\mathcal{D}$ and $\mathcal{D}^{\prime}$ are both cohomologous to $\mathcal{D}_{2}$, the second claim of Proposition 3.1 follows.

Remark 3.4 The second claim of Proposition 3.1 can probably be deduced from [27, Proposition 5.4] and some other statements in [27]. However, this would require a digression to "stable setting" which we avoid in this paper. For this reason, we decided to present a complete proof of Proposition 3.1 which is independent of any intermediate steps in [27].

\footnotetext{
12 Strictly speaking, only the homotopy class of the $\Lambda \mathrm{Lie}_{\infty}$-automorphism $U^{\mathcal{D}_{1}}$ is uniquely determined by $\mathcal{D}_{1}$.
} 


\section{Final remarks: connecting Drinfeld associators to the set of homotopy classes $\pi_{0}\left(V_{A} \rightsquigarrow C^{\bullet}(A)\right)$}

In this section we recall how to construct a $\mathrm{GRT}_{1}$-equivariant map $\mathfrak{B}$ from the set DrAssoc $_{1}$ of Drinfeld associators to the set

$$
\pi_{0}\left(\mathrm{Ger}_{\infty} \rightarrow \text { Braces }\right)
$$

of homotopy classes of operad morphisms (2.1) satisfying conditions (2.2).

Composing $\mathfrak{B}$ with the map $\mathfrak{T}$ (2.20), we get the desired map

$$
\mathfrak{T} \circ \mathfrak{B}: \operatorname{DrAssoc}_{1} \rightarrow \pi_{0}\left(V_{A} \rightsquigarrow C^{\bullet}(A)\right)
$$

from the set DrAssoc ${ }_{1}$ to the set of homotopy classes of $\Lambda$ Lie ${ }_{\infty}$-morphisms from $V_{A}$ to $C^{\bullet}(A)$ whose linear term is the Hochschild-Kostant-Rosenberg embedding.

Theorem 3.3 will then imply that map (4.1) is $\mathrm{GRT}_{1}$-equivariant.

\subsection{The sets DrAssoc ${ }_{\kappa}$ of Drinfeld associators}

In this short subsection, we briefly recall Drinfeld's associators and the Grothendieck-

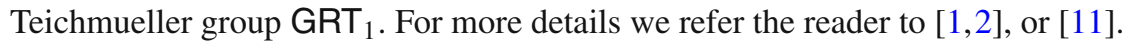

Let $m$ be an integer $\geq 2$. We denote by $\mathfrak{t}_{m}$ the Lie algebra generated by symbols $\left\{t^{i j}=t^{j i}\right\}_{1 \leq i \neq j \leq m}$ subject to the following relations:

$$
\begin{aligned}
{\left[t^{i j}, t^{i k}+t^{j k}\right] } & =0 & & \text { for any triple of distinct indices } i, j, k, \\
{\left[t^{i j}, t^{k l}\right] } & =0 & & \text { for any quadruple of distinct indices } i, j, k, l .
\end{aligned}
$$

The notation $A_{m}^{\mathrm{pb}}$ is reserved for the associative algebra (over $\mathbb{K}$ ) of formal power series in noncommutative symbols $\left\{t^{i j}=t^{j i}\right\}_{1 \leq i \neq j \leq m}$ subject to the same relations (4.2). Let us recall [25, Section 4] that the collection $A^{p b}:=\left\{A_{m}^{p b}\right\}_{m \geq 1}$ with $A_{1}^{p b}:=\mathbb{K}$ forms an operad in the category of associative $\mathbb{K}$-algebras.

Let $\mathfrak{l i}_{\mathfrak{e}}(x, y)$ be the degree completion of the free Lie algebra in two symbols $x$ and $y$ and let $\kappa$ be any element of $\mathbb{K}$.

The set $\operatorname{DrAssoc}_{\kappa}$ consists of elements $\Phi \in \exp (\operatorname{lie}(x, y))$ which satisfy the equations

$$
\begin{gathered}
\Phi(y, x) \Phi(x, y)=1, \\
\Phi\left(t^{12}, t^{23}+t^{24}\right) \Phi\left(t^{13}+t^{23}, t^{34}\right)=\Phi\left(t^{23}, t^{34}\right) \Phi\left(t^{12}+t^{13}, t^{24}+t^{34}\right) \Phi\left(t^{12}, t^{23}\right), \\
e^{\kappa\left(t^{13}+t^{23}\right) / 2}=\Phi\left(t^{13}, t^{12}\right) e^{\kappa t^{13} / 2} \Phi\left(t^{13}, t^{23}\right)^{-1} e^{\kappa t^{23} / 2} \Phi\left(t^{12}, t^{23}\right),
\end{gathered}
$$


and

$$
e^{\kappa\left(t^{12}+t^{13}\right) / 2}=\Phi\left(t^{23}, t^{13}\right)^{-1} e^{\kappa t^{13} / 2} \Phi\left(t^{12}, t^{13}\right) e^{\kappa t^{12} / 2} \Phi\left(t^{12}, t^{23}\right)^{-1}
$$

For $\kappa \neq 0$, elements $\Phi$ of DrAssoc ${ }_{\kappa}$ are called Drinfeld associators. However, for our purposes, we only need the set DrAssoc ${ }_{1}$ and the set DrAssoc . $_{0}$.

According to [11, Section 5], the set

$$
\operatorname{DrAssoc}_{0}
$$

forms a prounipotent group and, by [11, Proposition 5.5], this group acts simply transitively on the set of associators in DrAssoc 1 . Following [11], we denote the group DrAssoc $\operatorname{Db}_{0} \mathrm{GRT}_{1}$.

\subsection{A map $\mathfrak{B}$ from DrAssoc to $_{0} \pi_{0}\left(\mathrm{Ger}_{\infty} \rightarrow\right.$ Braces $)$}

Let us recall $[2,25]$ that collections of all braid groups can be assembled into the operad $\mathrm{PaB}$ in the category of $\mathbb{K}$-linear categories. Similarly, the collection of algebras $\left\{\mathrm{A}_{m}^{\mathrm{pb}}\right\}_{m \geq 1}$ can be "upgraded" to the operad PaCD also in the category of $\mathbb{K}$-linear categories. Every associator $\Phi \in$ DrAssoc $_{1}$ gives us an isomorphism of these operads

$$
I_{\Phi}: \mathrm{PaB} \stackrel{\cong}{\longrightarrow} \mathrm{PaCD} .
$$

The group $\mathrm{GRT}_{1}$ acts on the operad $\mathrm{PaCD}$ in such a way that, for every pair $g \in$ $\mathrm{GRT}_{1}, \quad \Phi \in$ DrAssoc $_{1}$, the diagram

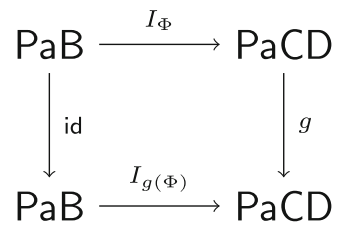

commutes.

Applying to $\mathrm{PaB}$ and $\mathrm{PaCD}$ the functor $C_{-} \bullet(, \mathbb{K})$, where $C_{\bullet}(, \mathbb{K})$ denotes the Hochschild chain complex with coefficients in $\mathbb{K}$, we get dg operads

$$
C_{-\bullet}(\mathrm{PaB}, \mathbb{K})
$$

and

$$
C_{-\bullet}(\mathrm{PaCD}, \mathbb{K}) \text {. }
$$


By naturality of $C_{-\bullet}(, \mathbb{K})$, diagram (4.9) gives us the commutative diagram

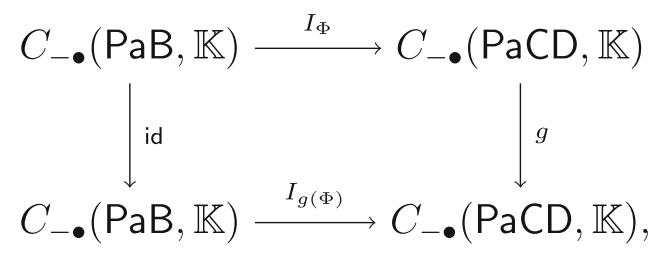

where, for simplicity, the maps corresponding to $I_{\Phi}, I_{g(\Phi)}$ and $g$ are denoted by the same letters, respectively.

Recall that Eq. (5) from [25] gives us the canonical quasi-isomorphism from the operad Ger to $C_{-}\left(\mathrm{A}^{\mathrm{pb}}, \mathbb{K}\right)$. The latter operad, in turn, receives the natural map

$$
C_{-\bullet}(\mathrm{PaCD}, \mathbb{K}) \rightarrow C_{-\bullet}\left(\mathrm{A}^{\mathrm{pb}}, \mathbb{K}\right)
$$

from $C_{-} \bullet(\mathrm{PaCD}, \mathbb{K})$ which is also known to be a quasi-isomorphism.

Thus, using the lifting property (see [5, Corollary 5.8]) for maps from the operad $\operatorname{Ger}_{\infty}=\operatorname{Cobar}\left(\operatorname{Ger}^{\vee}\right)$, we get the quasi-isomorphism ${ }^{13}$

$$
\operatorname{Ger}_{\infty} \stackrel{\sim}{\longrightarrow} C_{-} \cdot(\mathrm{PaCD}, \mathbb{K}) .
$$

Using this quasi-isomorphism and [5, Corollary 5.8], one can construct (see [27, Section 6.3.1]) a group homomorphism

$$
\mathrm{GRT}_{1} \rightarrow \exp (\mathfrak{g}),
$$

where the Lie algebra $\mathfrak{g}$ is defined in (3.4). By [27, Theorem 1.2], homomorphism (4.14) is an isomorphism.

Any specific solution of Deligne's conjecture on the Hochschild complex (see, for example, [4,8], or [21]) combined with Fiedorowicz's recognition principle [12] provides us with a sequence of quasi-isomorphisms

$$
\text { Braces } \stackrel{\sim}{\leftarrow} \stackrel{\sim}{\rightarrow} \bullet \stackrel{\sim}{\leftarrow} \cdot \cdots \bullet \stackrel{\sim}{\rightarrow} C_{-} \bullet(\mathrm{PaB}, \mathbb{K})
$$

which connects the dg operad Braces to $C_{-\bullet}(\mathrm{PaB}, \mathbb{K})$.

Hence, every associator $\Phi \in$ DrAssoc $_{1}$ gives us a sequence of quasi-isomorphisms

$$
\text { Braces } \stackrel{\sim}{\leftarrow} \stackrel{\sim}{\rightarrow} \bullet \stackrel{\sim}{\leftarrow} \bullet \cdots \bullet \stackrel{\sim}{\rightarrow} C_{-} \bullet(P a B, \mathbb{K}) \stackrel{I_{\Phi}}{\longrightarrow} C_{-} \cdot(\operatorname{PaCD}, \mathbb{K}) \stackrel{\sim}{\longleftarrow} \operatorname{Ger}_{\infty}
$$

connecting the dg operads Braces to $\mathrm{Ger}_{\infty}$.

\footnotetext{
13 By the same lifting property (see [5, Corollary 5.8]), we know that the homotopy type of the quasiisomorphism (4.13) is uniquely determined by the operad map Ger $\rightarrow C_{-\bullet}\left(\mathrm{A}^{\mathrm{pb}}, \mathbb{K}\right)$ from [25, Eq. (5)].
} 
Using [5, Corollary 5.8] once again, we conclude that the sequence of quasiisomorphisms (4.16) determines a unique homotopy class of quasi-isomorphisms (of dg operads)

$$
\Psi: \operatorname{Ger}_{\infty} \rightarrow \text { Braces. }
$$

Thus we get a well defined map

$$
\mathfrak{B}: \operatorname{DrAssoc}_{1} \rightarrow \pi_{0}\left(\text { Ger }_{\infty} \rightarrow \text { Braces }\right)
$$

In view of isomorphism (4.14), the set of homotopy classes $\pi_{0}$ (Ger G $_{\infty} \rightarrow$ Braces) is equipped with a natural action of $\mathrm{GRT}_{1}$. Moreover, the commutativity of diagram (4.12) implies that the map $\mathfrak{B}$ is $\mathrm{GRT}_{1}$-equivariant.

Thus, combining this observation with Theorem 3.3 we deduce the following corollary:

Corollary 4.1 Let $\pi_{0}\left(V_{A} \rightsquigarrow C^{\bullet}(A)\right)$ be the set of homotopy classes of $\Lambda$ Lie $_{\infty}$ quasi-isomorphisms which extend the Hochschild-Kostant-Rosenberg embedding of polyvector fields into Hochschild cochains. If we consider $\pi_{0}\left(V_{A} \rightsquigarrow C^{\bullet}(A)\right)$ as a set with the $\mathrm{GRT}_{1}$-action induced by isomorphism (4.14) then the composition

$$
\mathfrak{T} \circ \mathfrak{B}: \operatorname{DrAssoc}_{1} \rightarrow \pi_{0}\left(V_{A} \rightsquigarrow C^{\bullet}(A)\right)
$$

is $\mathrm{GRT}_{1}$-equivariant.

Remark 4.2 Any sequence of quasi-isomorphisms of dg operads (4.15) gives us an isomorphism between the objects corresponding to $C_{-}$( $(\mathrm{PaB}, \mathbb{K})$ and Braces in the homotopy category of $\mathrm{dg}$ operads. However, there is no reason to expect that different solutions of the Deligne conjecture give the same isomorphisms from $C_{-\bullet}(\mathrm{PaB}, \mathbb{K})$ to Braces in the homotopy category. Hence the resulting composition in (4.19) may depend on the choice of a specific solution of Deligne's conjecture on the Hochschild complex.

Acknowledgments We would like to thank Thomas Willwacher for useful discussions. We acknowledge the NSF Grant DMS-1161867 for a partial support. Finally, we would like to thank the anonymous referee for useful suggestions.

\section{Appendix A: Filtered $\Lambda^{-1}$ Lie $_{\infty}$-algebras}

Let $L$ be a cochain complex with the differential $\partial$. Recall that a $\Lambda^{-1}$ Lie $_{\infty}$-structure on $L$ is a sequence of degree 1 multi-brackets

$$
\{,, \ldots,\}_{m}: S^{m}(L) \rightarrow L, \quad m \geq 2
$$


satisfying the relations

$$
\begin{aligned}
& \partial\left\{v_{1}, v_{2}, \ldots, v_{m}\right\}+\sum_{i=1}^{m}(-1)^{\left|v_{1}\right|+\cdots+\left|v_{i-1}\right|}\left\{v_{1}, \ldots, v_{i-1}, \partial v_{i}, v_{i+1}, \ldots, v_{m}\right\} \\
& +\sum_{k=2}^{m-1} \sum_{\sigma \in \mathrm{Sh}_{k, m-k}}(-1)^{\varepsilon\left(\sigma ; v_{1}, \ldots, v_{m}\right)}\left\{\left\{v_{\sigma(1)}, \ldots, v_{\sigma(k)}\right\}, v_{\sigma(k+1)}, \ldots, v_{\sigma(m)}\right\}=0,
\end{aligned}
$$

where $(-1)^{\varepsilon\left(\sigma ; v_{1}, \ldots, v_{m}\right)}$ is the Koszul sign factor [see Eq. (1.1)].

We say that a $\Lambda^{-1}$ Lie $_{\infty}$-algebra $L$ is filtered if it is equipped with a complete descending filtration

$$
L=\mathcal{F}_{1} L \supset \mathcal{F}_{2} L \supset \mathcal{F}_{3} L \supset \ldots
$$

For such filtered $\Lambda^{-1}$ Lie $_{\infty}$-algebras we may define a Maurer-Cartan element as a degree zero element $\alpha$ satisfying the equation

$$
\partial \alpha+\sum_{m \geq 2} \frac{1}{m !}\{\alpha, \alpha, \ldots, \alpha\}_{m}=0
$$

Note that this equation makes sense for any degree 0 element $\alpha$ because $L=\mathcal{F}_{1} L$ and $L$ is complete with respect to filtration (5.3). Let us denote by $\operatorname{MC}(L)$ the set of Maurer-Cartan elements of a filtered $\Lambda^{-1}$ Lie $_{\infty}$-algebra $L$.

According to ${ }^{14}[14]$, the set $\mathrm{MC}(L)$ can be upgraded to an $\infty$-groupoid $\mathfrak{M C}(L)$ (i.e. a simplicial set satisfying the Kan condition). To introduce the $\infty$-groupoid $\mathfrak{M C}(L)$, we denote by $\Omega^{\bullet}\left(\Delta_{n}\right)$ the dg commutative $\mathbb{K}$-algebra of polynomial forms [14, Section 3] on the $n$-th geometric simplex $\Delta_{n}$. Next, we declare that set of $n$-simplices of $\mathfrak{M C}(L)$ is

$$
\operatorname{MC}\left(L \hat{\otimes} \Omega^{\bullet}\left(\Delta_{n}\right)\right),
$$

where $L$ is considered with the topology coming from filtration (5.3) and $\Omega^{\bullet}\left(\Delta_{n}\right)$ is considered with the discrete topology. The structure of the simplicial set is induced from the structure of a simplicial set on the sequence $\left\{\Omega^{\bullet}\left(\Delta_{n}\right)\right\}_{n \geq 0}$.

For example, 0-cells of $\mathfrak{M C}(L)$ are precisely Maurer-Cartan elements of $L$ and 1-cells are sums

$$
\alpha^{\prime}+d t \alpha^{\prime \prime}, \quad \alpha^{\prime} \in L^{0} \hat{\otimes} \mathbb{K}[t], \quad \alpha^{\prime \prime} \in L^{-1} \hat{\otimes} \mathbb{K}[t]
$$

\footnotetext{
14 A version of the Deligne-Getzler-Hinich $\infty$-groupoid for pro-nilpotent $\Lambda^{-1}$ Lie $_{\infty}$-algebras is introduced in [6, Section 4].
} 
satisfying the pair of equations

$$
\begin{aligned}
& \partial \alpha^{\prime}+\sum_{m \geq 2} \frac{1}{m !}\left\{\alpha^{\prime}, \alpha^{\prime}, \ldots, \alpha^{\prime}\right\}_{m}=0 \\
& \frac{d}{d t} \alpha^{\prime}=\partial \alpha^{\prime \prime}+\sum_{m \geq 1} \frac{1}{m !}\left\{\alpha^{\prime}, \alpha^{\prime}, \ldots, \alpha^{\prime}, \alpha^{\prime \prime}\right\}_{m+1}
\end{aligned}
$$

Thus, two 0-cells $\alpha_{0}, \alpha_{1}$ of $\mathfrak{M C}(L)$ (i.e. Maurer-Cartan elements of $L$ ) are isomorphic if there exists an element (5.6) satisfying (5.7) and (5.8) and such that

$$
\alpha_{0}=\left.\alpha^{\prime}\right|_{t=0} \text { and } \quad \alpha_{1}=\left.\alpha^{\prime}\right|_{t=1}
$$

We say that a 1-cell (5.6) connects $\alpha_{0}$ and $\alpha_{1}$.

\section{A.1: A lemma on adjusting Maurer-Cartan elements}

Let $\alpha$ be a Maurer-Cartan element of a filtered $\Lambda^{-1}$ Lie $_{\infty}$-algebra and $\xi$ be a degree -1 element in $\mathcal{F}_{n} L$ for some integer $n \geq 1$.

Let us consider the following sequence $\left\{\alpha_{k}^{\prime}\right\}_{k \geq 0}$ of degree zero elements in $L \hat{\otimes} \mathbb{K}[t]$

$$
\alpha_{0}^{\prime}:=\alpha, \quad \alpha_{k+1}^{\prime}(t):=\alpha+\int_{0}^{t} d t_{1}\left(\partial \xi+\sum_{m \geq 1} \frac{1}{m !}\left\{\alpha_{k}^{\prime}\left(t_{1}\right), \ldots, \alpha_{k}^{\prime}\left(t_{1}\right), \xi\right\}_{m+1}\right) .
$$

Since $L$ is complete with respect to filtration (5.3), the sequence $\left\{\alpha_{k}^{\prime}\right\}_{k \geq 0}$ convergences to a (degree 0 ) element $\alpha^{\prime} \in L \hat{\otimes} \mathbb{K}[t]$ which satisfies the integral equation

$$
\alpha^{\prime}(t)=\alpha+\int_{0}^{t} d t_{1}\left(\partial \xi+\sum_{m \geq 1} \frac{1}{m !}\left\{\alpha^{\prime}\left(t_{1}\right), \ldots, \alpha^{\prime}\left(t_{1}\right), \xi\right\}_{m+1}\right)
$$

We claim that

Lemma 5.1 If, as above, $\xi$ is a degree -1 element in $\mathcal{F}_{n} L$ and $\alpha^{\prime}$ is an element of $L \hat{\otimes} \mathbb{K}[t]$ obtained by recursive procedure (5.10) then the sum

$$
\alpha^{\prime}+d t \xi
$$

is a 1-cell of $\mathfrak{M C}(L)$ which connects $\alpha$ to another Maurer-Cartan element $\widetilde{\alpha}$ of $L$ such that

$$
\alpha^{\prime}-\alpha \in \mathcal{F}_{n} L \hat{\otimes} \mathbb{K}[t],
$$


and

$$
\widetilde{\alpha}-\alpha-\partial \xi \in \mathcal{F}_{n+1} L
$$

If the element $\xi$ satisfies the additional condition

$$
\partial \xi \in \mathcal{F}_{n+1} L
$$

then

$$
\alpha^{\prime}-\alpha \in \mathcal{F}_{n+1} L \hat{\otimes} \mathbb{K}[t],
$$

and

$$
\widetilde{\alpha}-\alpha-\partial \xi-\{\alpha, \xi\} \in \mathcal{F}_{n+2} L
$$

Proof Equation (5.11) implies that $\alpha^{\prime}$ satisfies the differential equation

$$
\frac{d}{d t} \alpha^{\prime}=\partial \xi+\sum_{m \geq 1} \frac{1}{m !}\left\{\alpha^{\prime}, \ldots, \alpha^{\prime}, \xi\right\}_{m+1}
$$

with the initial condition

$$
\left.\alpha^{\prime}\right|_{t=0}=\alpha
$$

Let us denote by $\Xi$ the following degree 1 element of $L \hat{\otimes} \mathbb{K}[t]$

$$
\Xi:=\partial \alpha^{\prime}+\sum_{m \geq 2} \frac{1}{m !}\left\{\alpha^{\prime}, \alpha^{\prime}, \ldots, \alpha^{\prime}\right\}_{m}
$$

A direct computation shows that $\Xi$ satisfies the following differential equation

$$
\frac{d}{d t} \Xi=-\sum_{m \geq 0} \frac{1}{m !}\left\{\alpha^{\prime}, \ldots, \alpha^{\prime}, \Xi, \xi\right\}_{m+2}
$$

Furthermore, since $\alpha$ is a Maurer-Cartan element of $L$, the element $\Xi$ satisfies the condition

$$
\left.\Xi\right|_{t=0}=0
$$

and hence $\Xi$ satisfies the integral equation

$$
\Xi(t)=-\int_{0}^{t} d t_{1}\left(\sum_{m \geq 0} \frac{1}{m !}\left\{\alpha^{\prime}\left(t_{1}\right), \ldots, \alpha^{\prime}\left(t_{1}\right), \Xi\left(t_{1}\right), \xi\right\}_{m+2}\right) .
$$


Equation (5.22) implies that

$$
\Xi \in \bigcap_{n \geq 1} \mathcal{F}_{n} L \hat{\otimes} \mathbb{K}[t]
$$

Therefore $\Xi=0$ and hence the limiting element $\alpha^{\prime}$ of sequence (5.10) is a MaurerCartan element of $L \hat{\otimes} \mathbb{K}[t]$.

Combining this observation with differential equation (5.18), we conclude that the element $\alpha^{\prime}+d t \xi \in L \hat{\otimes} \Omega^{\bullet}\left(\Delta_{1}\right)$ is indeed a 1-cell in $\mathfrak{M C}(L)$ which connects the Maurer-Cartan element $\alpha$ to the Maurer-Cartan element

$$
\widetilde{\alpha}:=\alpha+\int_{0}^{1} d t\left(\partial \xi+\sum_{m \geq 1} \frac{1}{m !}\left\{\alpha^{\prime}(t), \ldots, \alpha^{\prime}(t), \xi\right\}_{m+1}\right)
$$

Since $\xi \in \mathcal{F}_{n} L$ and $L=\mathcal{F}_{1} L$, equation (5.11) implies that

$$
\alpha^{\prime}-\alpha \in \mathcal{F}_{n} L \hat{\otimes} \mathbb{K}[t]
$$

and equation (5.23) implies that

$$
\widetilde{\alpha}-\alpha-\partial \xi \in \mathcal{F}_{n+1} L \text {. }
$$

Thus, the first part of Lemma 5.1 is proved.

If $\xi \in \mathcal{F}_{n} L$ and $\partial \xi \in \mathcal{F}_{n+1} L$ then, again, it is clear from (5.11) that inclusion (5.16) holds.

Finally, using inclusion (5.16) and equation (5.23), it is easy to see that

$$
\widetilde{\alpha}-\alpha-\partial \xi-\{\alpha, \xi\} \in \mathcal{F}_{n+2} L
$$

Lemma 5.1 is proved.

\section{A.2: Convolution $\Lambda^{-1}$ Lie $_{\infty}$-algebra, $\infty$-morphisms and their homotopies}

Let $\mathcal{C}$ be a coaugmented cooperad (in the category of graded vector spaces) satisfying the additional condition

$$
\mathcal{C}(0)=\mathbf{0}
$$

and $V$ be a cochain complex. (In this paper, $\mathcal{C}$ is usually the cooperad $\mathrm{Ger}^{\vee}$ ).

Following [7], we say that $V$ is a homotopy algebra of type $\mathcal{C}$ if $V$ carries $\operatorname{Cobar}(\mathcal{C})$ algebra structure or equivalently the $\mathcal{C}$-coalgebra 
has a degree 1 coderivation $Q$ satisfying

$$
\left.Q\right|_{V}=0
$$

and the Maurer-Cartan equation

$$
\left[d_{V}, Q\right]+\frac{1}{2}[Q, Q]=0
$$

where $d_{V}$ is the differential on $\mathcal{C}(V)$ induced from the one on $V$.

For two homotopy algebras $\left(V, Q_{V}\right)$ and $\left(W, Q_{W}\right)$ of type $\mathcal{C}$, we consider the graded vector space

$$
\operatorname{Hom}(\mathcal{C}(V), W)
$$

with the differential $\partial$

$$
\partial(f):=d_{W} \circ f-(-1)^{|f|} f \circ\left(d_{V}+Q_{V}\right)
$$

and the multi-brackets (of degree 1)

$$
\begin{gathered}
\{,, \ldots,\}_{m}: S^{m}(\operatorname{Hom}(\mathcal{C}(V), W)) \rightarrow \operatorname{Hom}(\mathcal{C}(V), W), \quad m \geq 2 \\
\left\{f_{1}, \ldots, f_{m}\right\}(X)=p_{W} \circ Q_{W}\left(1 \otimes f_{1} \otimes \cdots \otimes f_{m}\left(\Delta_{m}(X)\right)\right),
\end{gathered}
$$

where $\Delta_{m}$ is the $m$-th component of the comultiplication

$$
\Delta_{m}: \mathcal{C}(V) \rightarrow\left(\mathcal{C}(m) \otimes \mathcal{C}(V)^{\otimes m}\right)^{S_{m}}
$$

and $p_{W}$ is the canonical projection

$$
p_{W}: \mathcal{C}(W) \rightarrow W
$$

According to [7] or [10, Section 1.3], Eq. (5.27) define a $\Lambda^{-1}$ Lie $_{\infty}$-structure on the cochain complex $\operatorname{Hom}(\mathcal{C}(V), W)$ with the differential $\partial$ (5.26). The $\Lambda^{-1}$ Lie $_{\infty}$-algebra

$$
\operatorname{Hom}(\mathcal{C}(V), W)
$$

is called the convolution $\Lambda^{-1}$ Lie $_{\infty}$-algebra of the pair $V, W$.

The convolution $\Lambda^{-1}$ Lie $_{\infty}$-algebra $\operatorname{Hom}(\mathcal{C}(V), W)$ carries the obvious descending filtration "by arity"

$$
\mathcal{F}_{n} \operatorname{Hom}(\mathcal{C}(V), W)=\left\{f \in \operatorname{Hom}(\mathcal{C}(V), W)|f|_{\mathcal{C}(m) \otimes_{S_{m}} V^{\otimes m}}=0 \forall m<n\right\}
$$


$\operatorname{Hom}(\mathcal{C}(V), W)$ is obviously complete with respect to this filtration and

$$
\operatorname{Hom}(\mathcal{C}(V), W)=\mathcal{F}_{1} \operatorname{Hom}(\mathcal{C}(V), W)
$$

due to condition (5.24). In other words, under our assumption on the cooperad $\mathcal{C}$, the convolution $\Lambda^{-1}$ Lie $_{\infty}$-algebra $\operatorname{Hom}(\mathcal{C}(V), W)$ is pronilpotent.

According to [10, Proposition 3], $\infty$-morphisms from $V$ to $W$ are in bijection with Maurer-Cartan elements of $\operatorname{Hom}(\mathcal{C}(V), W)$ i.e. 0-cells of the Deligne-Getzler-Hinich $\infty$-groupoid corresponding to $\operatorname{Hom}(\mathcal{C}(V), W)$. Furthermore, due to [10, Corollary 2], two $\infty$-morphisms from $V$ to $W$ are homotopic if and only if the corresponding Maurer-Cartan elements are isomorphic 0-cells in the Deligne-Getzler-Hinich $\infty$ groupoid of $\operatorname{Hom}(\mathcal{C}(V), W)$.

\section{Appendix B: Tamarkin's rigidity}

Let $V_{A}$ denote the Gerstenhaber algebra of polyvector fields on the graded affine space corresponding to $A=\mathbb{K}\left[x^{1}, x^{2}, \ldots, x^{d}\right]$ with

$$
\left|x^{i}\right|=t_{i}
$$

As the graded commutative algebra over $\mathbb{K}, V_{A}$ is freely generated by variables

$$
x^{1}, x^{2}, \ldots, x^{d}, \theta_{1}, \theta_{2}, \ldots, \theta_{d}
$$

where $\theta_{i}$ carries degree $1-t_{i}$.

$$
V_{A}=\mathbb{K}\left[x^{1}, x^{2}, \ldots, x^{d}, \theta_{1}, \theta_{2}, \ldots, \theta_{d}\right]
$$

Let us denote by $\mu_{\wedge}$ and $\mu_{\{,\}}$the vectors in End $_{V_{A}}(2)$ corresponding to the multiplication and the Schouten bracket $\{$, $\}$ on $V_{A}$, respectively.

The composition of the canonical quasi-isomorphism

$$
\operatorname{Cobar}\left(\operatorname{Ger}^{\vee}\right) \rightarrow \text { Ger }
$$

and the map Ger $\rightarrow$ End $_{V_{A}}$ corresponds to the following Maurer-Cartan element

$$
\alpha:=\mu_{\wedge} \otimes\left\{b_{1}, b_{2}\right\}+\mu_{\{,\}} \otimes b_{1} b_{2}
$$

in the graded Lie algebra

$$
\operatorname{Conv}^{\oplus}\left(\operatorname{Ger}^{\vee}, \operatorname{End}_{V_{A}}\right):=\bigoplus_{n \geq 1} \operatorname{Hom}_{S_{n}}\left(\operatorname{Ger}^{\vee}(n), \operatorname{End}_{V_{A}}(n)\right)
$$


for which we frequently use the obvious identification ${ }^{15}$

$$
\operatorname{Conv}^{\oplus}\left(\operatorname{Ger}^{\vee}, \operatorname{End}_{V_{A}}\right) \cong \bigoplus_{n \geq 1}\left(\operatorname{End}_{V_{A}}(n) \otimes \Lambda^{-2} \operatorname{Ger}(n)\right)^{S_{n}}
$$

In this section, we consider $\mathrm{Conv}^{\oplus}\left(\mathrm{Ger}^{\vee}, \mathrm{End}_{V_{A}}\right)$ as the cochain complex with the following differential

$$
\partial:=[\alpha,]
$$

We observe that $\operatorname{Conv}^{\oplus}\left(\mathrm{Ger}^{\vee}\right.$, End $\left._{V_{A}}\right)$ carries the natural descending filtration "by arity":

$$
\begin{aligned}
\operatorname{Conv}^{\oplus}\left(\operatorname{Ger}^{\vee}, \text { End }_{V_{A}}\right) & =\mathcal{F}_{0} \operatorname{Conv}^{\oplus}\left(\operatorname{Ger}^{\vee}, \operatorname{End}_{V_{A}}\right) \supset \mathcal{F}_{1} \operatorname{Conv}^{\oplus}\left(\operatorname{Ger}^{\vee}, \text { End }_{V_{A}}\right) \supset \cdots \\
\mathcal{F}_{m} \operatorname{Conv}^{\oplus}\left(\operatorname{Ger}^{\vee}, \operatorname{End}_{V_{A}}\right) & :=\bigoplus_{n \geq m+1}\left(\operatorname{End}_{V_{A}}(n) \otimes \Lambda^{-2} \operatorname{Ger}(n)\right)^{S_{n}} .
\end{aligned}
$$

More precisely,

$$
\partial\left(\operatorname{End}_{V_{A}}(n) \otimes \Lambda^{-2} \operatorname{Ger}(n)\right)^{S_{n}} \subset\left(\operatorname{End}_{V_{A}}(n+1) \otimes \Lambda^{-2} \operatorname{Ger}(n+1)\right)^{S_{n+1}} .
$$

In particular, every cocycle $X \in \mathrm{Conv}^{\oplus}\left(\mathrm{Ger}^{\vee}, \mathrm{End}_{V_{A}}\right)$ is a finite sum

$$
X=\sum_{n \geq 1} X_{n}, \quad X_{n} \in\left(\operatorname{End}_{V_{A}}(n) \otimes \Lambda^{-2} \operatorname{Ger}(n)\right)^{S_{n}}
$$

where each individual term $X_{n}$ is a cocycle.

In this paper, we need the following version of Tamarkin's rigidity

Theorem 6.1 If $n$ is an integer $\geq 2$ then for every cocycle

$$
X \in\left(\operatorname{End}_{V_{A}}(n) \otimes \Lambda^{-2} \operatorname{Ger}(n)\right)^{S_{n}} \subset \operatorname{Conv}^{\oplus}\left(\operatorname{Ger}^{\vee}, \operatorname{End}_{V_{A}}\right)
$$

there exists a cochain $Y \in\left(\operatorname{End}_{V_{A}}(n-1) \otimes \Lambda^{-2} \operatorname{Ger}(n-1)\right)^{S_{n-1}}$ such that

$$
X=\partial Y \text {. }
$$

Remark 6.2 Note that the above statement is different from Tamarkin's rigidity in the "stable setting" [5, Section 12]. According to [5, Corollary 12.2], one may think that

\footnotetext{
$\overline{15}$ Recall that the cooperad $\mathrm{Ger}^{\vee}$ is the linear dual of the operad $\Lambda^{-2}$ Ger.
} 
the vector

$$
\mu_{\{,\}} \otimes b_{1} b_{2}
$$

is a non-trivial cocycle in (6.3). In fact,

$$
\mu_{\{,\}} \otimes b_{1} b_{2}=\left[\alpha, P \otimes b_{1}\right]
$$

where $P$ is the following version of the "Euler derivation" of $V_{A}$.

$$
P(v):=\sum_{i=1}^{d} \theta_{i} \frac{\partial}{\partial \theta_{i}}
$$

Proof of Theorem 6.1 Theorem 6.1 is only a slight generalization of the statement proved in Section 5.4 of [15] and, in the proof given here, we pretty much follow the same line of arguments as in [15, Section 5.4].

First, we introduce an additional set of auxiliary variables

$$
\check{x}_{1}, \check{x}_{2}, \ldots, \check{x}_{d}, \check{\theta}^{1}, \check{\theta}^{2}, \ldots, \check{\theta}^{d}
$$

of degrees

$$
\left|\check{x}_{i}\right|=2-t_{i}, \quad\left|\check{\theta}^{i}\right|=t_{i}+1
$$

Second, we consider the de Rham complex of $V_{A}$ :

$$
\Omega_{\mathbb{K}}^{\bullet} V_{A}:=V_{A}\left[\check{x}_{1}, \check{x}_{2}, \ldots, \check{x}_{d}, \check{\theta}_{1}, \check{\theta}_{2}, \ldots, \check{\theta}_{d}\right]
$$

with the differential

$$
D=\sum_{i=1}^{d} \check{x}_{i} \frac{\partial}{\partial \theta_{i}}+\sum_{i=1}^{d} \check{\theta}^{i} \frac{\partial}{\partial x^{i}}
$$

and equip it with the following descending filtration:

$$
\begin{aligned}
& \mathcal{F}_{m} \Omega_{\mathbb{K}}^{\bullet} V_{A}:=\left\{P \in V_{A}\left[\check{x}_{1}, \check{x}_{2}, \ldots, \check{x}_{d}, \check{\theta}_{1}, \check{\theta}_{2}, \ldots, \check{\theta}_{d}\right]\right. \\
& \left.\quad \text { the total degree of } P \text { in } \check{x}_{1}, \ldots, \check{x}_{d}, \check{\theta}_{1}, \ldots, \check{\theta}_{d} \text { is } \geq m+1\right\} .
\end{aligned}
$$

Next, we observe that every homogeneous vector ${ }^{16}$

$$
P=P_{j_{1} j_{2} \ldots j_{q}}^{i_{1} i_{2} \ldots i_{k}} \check{x}_{i_{1}} \ldots \check{x}_{i_{k}} \check{\theta}^{j_{1}} \ldots \check{\theta}^{j_{q}} \in V_{A}\left[\check{x}_{1}, \check{x}_{2}, \ldots, \check{x}_{d}, \check{\theta}_{1}, \check{\theta}_{2}, \ldots, \check{\theta}_{d}\right]
$$

16 Summation over repeated indices is assumed. 
defines an element $P^{\text {End }} \in \operatorname{End}_{V_{A}}(k+q)$ :

$$
\begin{aligned}
P^{\text {End }}\left(v_{1}, v_{2}, \ldots, v_{k+q}\right):= & \sum_{\sigma \in S_{k+q}} \pm P_{j_{1} j_{2} \ldots j_{q}}^{i_{1} i_{2} \ldots i_{k}} \partial_{x_{1}{ }_{1}} v_{\sigma(1)} \partial_{x^{i_{2}}} v_{\sigma(2)} \ldots \partial_{x^{i_{k}}} v_{\sigma(k)} \\
& \times \partial_{\theta_{j_{1}}} v_{\sigma(k+1)} \partial_{\theta_{j_{2}}} v_{\sigma(k+2)} \ldots \partial_{\theta_{j q}} v_{\sigma(k+q)},
\end{aligned}
$$

where the sign factors \pm are determined by the usual Koszul rule.

Finally, we claim that the formula

$$
\mathrm{VH}(P):=P^{\mathrm{End}} \otimes b_{1} b_{2} \ldots b_{k+q}
$$

defines a degree zero injective map

$$
\mathrm{VH}: \mathbf{s}^{-2} \mathcal{F}_{0} \Omega_{\mathbb{K}}^{\bullet} V_{A} \rightarrow \operatorname{Conv}^{\oplus}\left(\mathrm{Ger}^{\vee}, \text { End }_{V_{A}}\right)
$$

which is compatible with filtrations (6.6) and (6.12).

A direct computation shows that $\mathrm{VH}$ intertwines differentials (6.5) and (6.11).

Let $m$ be an integer and

$$
\mathcal{G}^{m} \operatorname{Conv}^{\oplus}\left(\mathrm{Ger}^{\vee}, \text { End }_{V_{A}}\right)
$$

be the subspace of $\operatorname{Conv}^{\oplus}\left(\mathrm{Ger}^{\vee}, \mathrm{End}_{V_{A}}\right)$ of sums

$$
\sum_{i} M_{i} \otimes q_{i} \in \bigoplus_{n \geq 1}\left(\operatorname{End}_{V_{A}}(n) \otimes \Lambda^{-2} \operatorname{Ger}(n)\right)^{S_{n}}
$$

satisfying the condition

$$
\text { the number of Lie brackets in } q_{i}-\left|M_{i} \otimes q_{i}\right| \leq m \text {. }
$$

It is easy to see that the sequence of subspaces (6.16)

$$
\begin{aligned}
& \ldots \subset \mathcal{G}^{-1} \operatorname{Conv}^{\oplus}\left(\mathrm{Ger}^{\vee}, \text { End }_{V_{A}}\right) \subset \mathcal{G}^{0} \operatorname{Conv}^{\oplus}\left(\text { Ger }^{\vee}, \text { End }_{V_{A}}\right) \\
& \subset \mathcal{G}^{1} \operatorname{Conv}^{\oplus}\left(\operatorname{Ger}^{\vee}, \text { End }_{V_{A}}\right) \subset \ldots
\end{aligned}
$$

form an ascending filtration on the cochain complex $\operatorname{Conv}^{\oplus}\left(\operatorname{Ger}^{\vee}, \operatorname{End}_{V_{A}}\right)$ and the associated graded cochain complex

$$
\mathrm{Gr}_{\mathcal{G}} \operatorname{Conv}^{\oplus}\left(\mathrm{Ger}^{\vee}, \mathrm{End}_{V_{A}}\right)
$$

is isomorphic to

$$
\bigoplus_{n \geq 1}\left(\operatorname{End}_{V_{A}}(n) \otimes \Lambda^{-2} \operatorname{Ger}(n)\right)^{S_{n}}
$$


with the differential

$$
\partial^{\mathrm{Gr}}=\left[\mu_{\wedge} \otimes\left\{b_{1}, b_{2}\right\}, \quad\right]
$$

where $\mu_{\wedge}$ is the vector in $\mathrm{End}_{V_{A}}$ (2) which corresponds to the multiplication on $V_{A}$.

Let us observe that (6.19) is naturally a $V_{A}$-module (where $V_{A}$ is viewed as the graded commutative algebra), differential (6.20) is $V_{A}$-linear, and since

$$
\operatorname{Ger}^{\vee}\left(V_{A}\right)=\Lambda^{2} \operatorname{coCom}\left(\Lambda \operatorname{coLie}\left(V_{A}\right)\right),
$$

cochain complex (6.19) is isomorphic to

$$
\operatorname{Hom}_{V_{A}}\left(\mathbf{s}^{2} \underline{S}_{V_{A}}\left(\mathbf{s}^{-1} V_{A} \otimes_{\mathbb{K}} \operatorname{coLie}\left(\mathbf{s}^{-1} V_{A}\right)\right), V_{A}\right)
$$

with the differential coming from the one on the Harrison homological ${ }^{17}$ complex [19, Section 4.2.10]

$$
V_{A} \otimes_{\mathbb{K}} \operatorname{coLie}\left(\mathbf{s}^{-1} V_{A}\right)
$$

of the graded commutative algebra $V_{A}$ with coefficients in $V_{A}$.

Since $V_{A}$ is freely generated by elements $x^{1}, \ldots, x^{d}, \theta_{1}, \ldots, \theta_{d}$, Theorem 3.5.6 and Proposition 4.2.11 from [19] imply that the embedding

$$
\begin{aligned}
& I_{\text {Harr }}: \bigoplus_{i=1}^{d} V_{A} e^{i} \oplus \bigoplus_{i=1}^{d} V_{A} f_{i} \rightarrow V_{A} \otimes \operatorname{coLie}\left(\mathbf{s}^{-1} V_{A}\right) \\
& I_{\text {Harr }}\left(e^{i}\right):=1 \otimes \mathbf{s}^{-1} x^{i}, \quad I_{\text {Harr }}\left(f_{i}\right):=1 \otimes \mathbf{s}^{-1} \theta_{i}
\end{aligned}
$$

from the free $V_{A}$-module

$$
\bigoplus_{i=1}^{d} V_{A} e^{i} \oplus \bigoplus_{i=1}^{d} V_{A} f_{i}, \quad\left|e^{i}\right|:=t_{i}-1, \quad\left|f_{i}\right|:=-t_{i}
$$

is a quasi-isomorphism of cochain complexes of $V_{A}$-modules from (6.24) with the zero differential to (6.22) with the Harrison differential.

Since (6.23) is a quasi-isomorphism of cochain complexes of free $V_{A}$-modules, it induces a quasi-isomorphism of cochain complexes of (free) $V_{A}$-modules:

$$
\mathbf{s}^{2} V_{A}\left[\mathbf{s}^{-1} e^{1}, \ldots, \mathbf{s}^{-1} e^{d}, \mathbf{s}^{-1} f_{1}, \ldots, \mathbf{s}^{-1} f_{d}\right] \rightarrow \mathbf{s}^{2} S_{V_{A}}\left(\mathbf{s}^{-1} V_{A} \otimes_{\mathbb{K}} \operatorname{coLie}\left(\mathbf{s}^{-1} V_{A}\right)\right),
$$

where the source carries the zero differential.

\footnotetext{
17 The cochain complex in (6.22) is obtained from the conventional Harrison homological complex from [19, Section 4.2 .10$]$ by reversing the grading.
} 
Therefore, map (6.15) induces a quasi-isomorphism of cochain complexes

$$
\mathbf{s}^{-2} \mathcal{F}_{0} \Omega_{\mathbb{K}}^{\bullet} V_{A} \rightarrow \operatorname{Gr}_{\mathcal{G}} \operatorname{Conv}^{\oplus}\left(\text { Ger }^{\vee}, \text { End }_{V_{A}}\right),
$$

where the source is considered with the zero differential.

Thus, by Lemma A.3 from [5], map (6.15) is a quasi-isomorphism of cochain complexes.

Let $n \geq 2$ and

$$
X \in\left(\operatorname{End}_{V_{A}}(n) \otimes \Lambda^{-2} \operatorname{Ger}(n)\right)^{S_{n}} \subset \operatorname{Conv}^{\oplus}\left(\operatorname{Ger}^{\vee}, \operatorname{End}_{V_{A}}\right)
$$

be a cocycle.

Since (6.15) is a quasi-isomorphism of cochain complexes, there exists a cocycle

$$
\tilde{X} \in \mathbf{s}^{-2} \mathcal{F}_{0} \Omega_{\mathbb{K}}^{\bullet} V_{A}
$$

such that $X$ is cohomologous to $\mathrm{VH}(\tilde{X})$.

Let us observe that de Rham differential $D$ (6.11) satisfies the property

$$
D\left(\mathcal{F}_{0} \Omega_{\mathbb{K}}^{\bullet} V_{A}\right) \subset \mathcal{F}_{1} \Omega_{\mathbb{K}}^{\bullet} V_{A}
$$

Hence, since VH is injective, we conclude that

$$
\widetilde{X} \in \mathbf{s}^{-2} \mathcal{F}_{1} \Omega_{\mathbb{K}}^{\bullet} V_{A}
$$

It is obvious that every cocycle in $\mathcal{F}_{1} \Omega_{\mathbb{K}}^{\bullet} V_{A}$ is exact in $\mathcal{F}_{0} \Omega_{\mathbb{K}}^{\bullet} V_{A}$. Therefore $\tilde{X}$ is exact and so is cocycle (6.26).

Combining this statement with property (6.7) we easily deduce Theorem 6.1.

\section{B.1: The standard Gerstenhaber structure on $V_{A}$ is "rigid"}

The first consequence of Theorem 6.1 is the following corollary:

Corollary 6.3 Let $V_{A}$ be, as above, the algebra of polyvector fields on a graded affine space and $Q$ be a $\mathrm{Ger}_{\infty}$-structure on $V_{A}$ whose binary operations are the Schouten bracket and the usual multiplication. Then the identity map id : $V_{A} \rightarrow V_{A}$ can be extended to a $\mathrm{Ger}_{\infty}$ morphism

$$
U_{\text {corr }}: V_{A} \rightsquigarrow V_{A}^{Q}
$$

from $V_{A}$ with the standard Gerstenhaber structure to $V_{A}$ with the $\operatorname{Ger}_{\infty}$-structure $Q$.

Proof To prove this statement, we consider the graded space

$$
\operatorname{Hom}\left(\operatorname{Ger}^{\vee}\left(V_{A}\right), V_{A}\right)
$$


with two different algebraic structures. First, (6.30) is identified with the convolution Lie algebra ${ }^{18}$

$$
\operatorname{Conv}\left(\operatorname{Ger}^{\vee}, \text { End }_{V_{A}}\right)
$$

with the Lie bracket $[$, ] defined in terms of the binary (degree zero) operation $\bullet$ from [5, Section 4, Eq. (4.2)].

To introduce the second algebraic structure on (6.30), we recall that a Ger $_{\infty}$ structure on $V_{A}$ is precisely a degree 1 element

$$
Q=Q_{2}+\sum_{n \geq 3} Q_{n} \quad Q_{n} \in \operatorname{Hom}_{S_{n}}\left(\operatorname{Ger}^{\vee}(n) \otimes V_{A}^{\otimes n}, V_{A}\right)
$$

in (6.31) satisfying the Maurer-Cartan equation

$$
[Q, Q]=0
$$

and the above condition on the binary operations is equivalent to the requirement

$$
Q_{2}=\alpha,
$$

where $\alpha$ is Maurer-Cartan element (6.2) of (6.31).

Given such a $\operatorname{Ger}_{\infty}$-structure $Q$ on $V_{A}$, we get the convolution $\Lambda^{-1}$ Lie $_{\infty}$-algebra

$$
\operatorname{Hom}\left(\operatorname{Ger}^{\vee}\left(V_{A}\right), V_{A}^{Q}\right)
$$

corresponding to the pair $\left(V_{A}, V_{A}^{Q}\right)$, where the first entry $V_{A}$ is considered with the standard Gerstenhaber structure and the second entry is considered with the above Ger $_{\infty}$-structure $Q$.

As a graded vector space, $\Lambda^{-1}$ Lie $_{\infty}$-algebra (6.35) coincides with (6.30). However, it carries a non-zero differential $d_{\alpha}$ given by the formula

$$
d_{\alpha}(P)=-(-1)^{|P|} P \bullet \alpha
$$

and the corresponding (degree 1) brackets

$$
\{,, \ldots,\}_{k}: S^{k}\left(\operatorname{Hom}\left(\operatorname{Ger}^{\vee}\left(V_{A}\right), V_{A}^{Q}\right)\right) \rightarrow \operatorname{Hom}\left(\operatorname{Ger}^{\vee}\left(V_{A}\right), V_{A}^{Q}\right)
$$

are defined by general formula (5.27) in terms of the $\mathrm{Ger}^{\vee}$-coalgebra structure on $\operatorname{Ger}^{\vee}\left(V_{A}\right)$ and the $\operatorname{Ger}_{\infty}$-structure $Q$ on $V_{A}$.

18 In our case, Lie algebra (6.31) carries the zero differential. 
Let us recall $[7,10]$ that $\operatorname{Ger}_{\infty}$-morphisms from $V_{A}$ to $V_{A}^{Q}$ are in bijection with Maurer-Cartan elements ${ }^{19}$

$$
\beta=\sum_{n \geq 1} \beta_{n}, \quad \beta_{n} \in \operatorname{Hom}_{S_{n}}\left(\operatorname{Ger}^{\vee}(n) \otimes V_{A}^{\otimes n}, V_{A}\right)
$$

of $\Lambda^{-1}$ Lie $_{\infty}$-algebra (6.35) such that $\beta_{1}$ corresponds to the linear term of the corresponding Ger $_{\infty}$-morphism.

Thus our goal is to prove that, for every Maurer-Cartan element $Q$ (6.32) of Lie algebra (6.31) satisfying condition (6.34), there exists a Maurer-Cartan element $\beta$ (see (6.37)) of $\Lambda^{-1}$ Lie $_{\infty}$-algebra (6.35) such that

$$
\beta_{1}=\mathrm{id}: V_{A} \rightarrow V_{A}
$$

Condition (6.34) implies that the element

$$
\beta^{(1)}:=\mathrm{id} \in \operatorname{Hom}\left(\operatorname{Ger}^{\vee}\left(V_{A}\right), V_{A}^{Q}\right)
$$

satisfies the equation (in the $\Lambda^{-1} \operatorname{Lie}_{\infty}$-algebra $\operatorname{Hom}\left(\operatorname{Ger}^{\vee}\left(V_{A}\right), V_{A}^{Q}\right)$ )

$$
\left(d_{\alpha}\left(\beta^{(1)}\right)+\sum_{k \geq 2} \frac{1}{k !}\left\{\beta^{(1)}, \ldots, \beta^{(1)}\right\}_{k}\right)(X)=0
$$

for every $X \in\left(\operatorname{Ger}^{\vee}(m) \otimes V_{A}^{\otimes m}\right)_{S_{m}}$ with $m \leq 2$.

Let us assume that we constructed (by induction) a degree zero element

$$
\beta^{(n-1)}=\mathrm{id}+\beta_{2}+\beta_{3}+\cdots+\beta_{n-1}, \quad \beta_{j} \in \operatorname{Hom}_{S_{j}}\left(\operatorname{Ger}^{\vee}(j) \otimes V_{A}^{\otimes j}, V_{A}\right)
$$

such that

$$
\left(d_{\alpha}\left(\beta^{(n-1)}\right)+\sum_{k \geq 2} \frac{1}{k !}\left\{\beta^{(n-1)}, \ldots, \beta^{(n-1)}\right\}_{k}\right)(X)=0
$$

for every $X \in\left(\operatorname{Ger}^{\vee}(m) \otimes V_{A}^{\otimes m}\right)_{S_{m}}$ with $m \leq n$.

We will try to find an element

$$
\beta_{n} \in \operatorname{Hom}_{S_{n}}\left(\operatorname{Ger}^{\vee}(n) \otimes V_{A}^{\otimes n}, V_{A}\right)
$$

such that the sum

$$
\beta^{(n)}:=\mathrm{id}+\beta_{2}+\beta_{3}+\cdots+\beta_{n-1}+\beta_{n}
$$

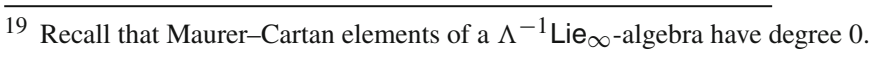


satisfies the equation

$$
\left(d_{\alpha}\left(\beta^{(n)}\right)+\sum_{k \geq 2} \frac{1}{k !}\left\{\beta^{(n)}, \ldots, \beta^{(n)}\right\}_{k}\right)(X)=0
$$

for every $X \in\left(\operatorname{Ger}^{\vee}(m) \otimes V_{A}^{\otimes m}\right)_{S_{m}}$ with $m \leq n+1$.

Since $\beta_{n} \in \operatorname{Hom}_{S_{n}}\left(\operatorname{Ger}^{\vee}(n) \otimes V_{A}^{\otimes n}, V_{A}\right)$ and (6.41) is satisfied for every $X \in$ $\left(\operatorname{Ger}^{\vee}(m) \otimes V_{A}^{\otimes m}\right)_{S_{m}}$ with $m \leq n$, equation (6.44) is also satisfied for every $X \in$ $\left(\operatorname{Ger}^{\vee}(m) \otimes V_{A}^{\otimes m}\right)_{S_{m}}$ with $m \leq n$.

For $X \in\left(\operatorname{Ger}^{\vee}(n+1) \otimes V_{A}^{\otimes(n+1)}\right)_{S_{n+1}}$, Eq. (6.44) can be rewritten as

$$
-\beta_{n} \bullet \alpha(X)+\alpha \bullet \beta_{n}(X)=-\sum_{k \geq 2} \frac{1}{k !}\left\{\beta^{(n-1)}, \ldots, \beta^{(n-1)}\right\}_{k}(X)
$$

Let us denote by $\gamma$ the element in $\operatorname{Hom}_{S_{n+1}}\left(\operatorname{Ger}^{\vee}(n+1) \otimes V_{A}^{\otimes(n+1)}, V_{A}\right)$ defined as

$$
\gamma:=\left.\sum_{k \geq 2} \frac{1}{k !}\left\{\beta^{(n-1)}, \ldots, \beta^{(n-1)}\right\}_{k}\right|_{\operatorname{Ger}^{\vee}(n+1) \otimes V_{A}^{\otimes(n+1)}}
$$

Evaluating the Bianchi type identity [14, Lemma 4.5]

$$
\begin{aligned}
& \sum_{k \geq 2} \frac{1}{k !} d_{\alpha}\left\{\beta^{(n-1)}, \ldots, \beta^{(n-1)}\right\}_{k}+\sum_{k \geq 1} \frac{1}{k !}\left\{\beta^{(n-1)}, \ldots, \beta^{(n-1)},\right. \\
& \left.d_{\alpha} \beta^{(n-1)}\right\}_{k+1}+\sum_{\substack{k \geq 2 \\
t \geq 1}} \frac{1}{k ! t !}\left\{\beta^{(n-1)}, \ldots, \beta^{(n-1)},\left\{\beta^{(n-1)}, \ldots, \beta^{(n-1)}\right\}_{k}\right\}_{t+1}=0
\end{aligned}
$$

on an arbitrary element

$$
Y \in\left(\operatorname{Ger}^{\vee}(n+2) \otimes V_{A}^{\otimes(n+2)}\right)_{S_{n+2}}
$$

and using the fact that

$$
\beta^{(n-1)}(X)=0, \quad \forall X \in\left(\operatorname{Ger}^{\vee}(m) \otimes V_{A}^{\otimes m}\right)_{S_{m}} \quad \text { with } \quad m \geq n
$$

we deduce that element $\gamma(6.46)$ is a cocycle in cochain complex (6.3) with differential (6.5).

Thus Theorem 6.1 implies that Eq. (6.45) can always be solved for $\beta_{n}$.

This inductive argument concludes the proof of Corollary 6.3. 


\section{B.2 The Gerstenhaber algebra $V_{A}$ is intrinsically formal}

Let $\left(C^{\bullet}, \mathfrak{d}\right)$ be an arbitrary cochain complex whose cohomology is isomorphic to $V_{A}$

$$
H^{\bullet}\left(C^{\bullet}\right) \cong V_{A}
$$

Let us consider $V_{A}$ as the cochain complex with the zero differential and choose $\mathrm{e}^{20}$ a quasi-isomorphism of cochain complexes

$$
I: V_{A} \rightarrow C^{\bullet}
$$

Let us assume that $C^{\bullet}$ carries a $\operatorname{Ger}_{\infty}$-structure such that the map $I$ induces an isomorphism of Gerstenhaber algebras $V_{A} \cong H^{\bullet}\left(C^{\bullet}\right)$.

Then Theorem 6.1 gives us the following remarkable corollary:

Corollary 6.4 There exists a Ger $_{\infty}$-morphism

$$
U: V_{A} \rightsquigarrow C^{\bullet}
$$

whose linear term coincides with I (6.49). Moreover, any two such $\mathrm{Ger}_{\infty}$-morphisms

$$
U, \widetilde{U}: V_{A} \rightsquigarrow C^{\bullet}
$$

are homotopy equivalent.

Remark 6.5 The above statement is a slight refinement of one proved in [15, Section 5]. Following Hinich, we say that the Gerstenhaber algebra $V_{A}$ is intrinsically formal.

Proof of Corollary 6.4 By the Homotopy Transfer Theorem [7, Section 5], [20, Section 10.3], there exists a $\operatorname{Ger}_{\infty}$-structure $Q$ on $V_{A}$ and a $\operatorname{Ger}_{\infty}$-quasi-isomorphism

$$
U^{\prime}: V_{A}^{Q} \rightsquigarrow C^{\bullet}
$$

such that

- the binary operations of the $\operatorname{Ger}_{\infty}$-structure $Q$ on $V_{A}$ are the Schouten bracket and the usual multiplication of polyvector fields,

- the linear term of $U^{\prime}$ coincides with $I$.

Corollary 6.3 implies that there exists a Ger $_{\infty}$-morphism

$$
U_{\text {corr }}: V_{A} \rightsquigarrow V_{A}^{Q},
$$

whose linear term is the identity map id : $V_{A} \rightarrow V_{A}$.

\footnotetext{
${ }^{20}$ Such a quasi-isomorphism exists since we are dealing with cochain complexes of vector spaces over a field.
} 
Hence the composition

$$
U=U^{\prime} \circ U_{\text {corr }}: V_{A} \rightsquigarrow C^{\bullet}
$$

is a desired Ger $_{\infty}$-morphism.

To prove the second claim, we need the $\Lambda^{-1}$ Lie $_{\infty}$-algebra

$$
\operatorname{Hom}\left(\operatorname{Ger}^{\vee}\left(V_{A}\right), C^{\bullet}\right)
$$

corresponding to the Gerstenhaber algebra $V_{A}$ and the $\operatorname{Ger}_{\infty}$-algebra $C^{\bullet}$. The differential $\mathcal{D}$ on (6.55) is given by the formula

$$
\mathcal{D}(\Psi):=\mathfrak{d} \circ \Psi-(-1)^{|\Psi|} \Psi \circ Q_{\wedge,\{,\}}, \quad \Psi \in \operatorname{Hom}\left(\operatorname{Ger}^{\vee}\left(V_{A}\right), C^{\bullet}\right),
$$

where $\mathfrak{d}$ is the differential on $C^{\bullet}$ and $Q_{\wedge,\{,\}}$ is the differential on the $\mathrm{Ger}^{\vee}$-coalgebra $\operatorname{Ger}^{\vee}\left(V_{A}\right)$ corresponding to the standard Gerstenhaber structure on $V_{A}$.

The multi-brackets $\{, \ldots,\}_{m}$ are defined by the general formula [see Eq. (5.27)] in terms of the $\operatorname{Ger}^{\vee}$-coalgebra structure on $\operatorname{Ger}^{\vee}\left(V_{A}\right)$ and the $\operatorname{Ger}_{\infty}$-structure on $C^{\bullet}$.

Let us recall (see Appendix A.2 for more details) that Ger $_{\infty}$-morphisms from $V_{A}$ to $C^{\bullet}$ are in bijection with Maurer-Cartan elements of $\Lambda^{-1}$ Lie $_{\infty}$-algebra (6.55) and Ger $_{\infty}$-morphisms (6.51) are homotopy equivalent if and only if the corresponding Maurer-Cartan elements $P$ and $\widetilde{P}$ in (6.55) are isomorphic 0-cells in the DeligneGetzler-Hinich $\infty$-groupoid [14] of (6.55).

So our goal is to prove that any two Maurer-Cartan elements $P$ and $\widetilde{P}$ in (6.55) satisfying

$$
\left.P\right|_{V_{A}}=\left.\widetilde{P}\right|_{V_{A}}=I: V_{A} \rightarrow C^{\bullet}
$$

are isomorphic.

Condition (6.57) implies that

$$
\widetilde{P}-P \in \mathcal{F}_{2} \operatorname{Hom}\left(\operatorname{Ger}^{\vee}\left(V_{A}\right), C^{\bullet}\right),
$$

where $\mathcal{F}_{\bullet} \operatorname{Hom}\left(\operatorname{Ger}^{\vee}\left(V_{A}\right), C^{\bullet}\right)$ is the arity filtration (5.29) on $\operatorname{Hom}\left(\operatorname{Ger}^{\vee}\left(V_{A}\right), C^{\bullet}\right)$.

Let us assume that we constructed a sequence of Maurer-Cartan elements

$$
P=P_{2}, P_{3}, P_{4}, \ldots, P_{n+1}
$$

such that for every $2 \leq m \leq n+1$

$$
\widetilde{P}-P_{m} \in \mathcal{F}_{m} \operatorname{Hom}\left(\operatorname{Ger}^{\vee}\left(V_{A}\right), C^{\bullet}\right)
$$

and for every $2 \leq m \leq n$ there exists 1-cell

$$
P_{m}^{\prime}(t)+d t \xi_{m-1} \in \operatorname{Hom}\left(\operatorname{Ger}^{\vee}\left(V_{A}\right), C^{\bullet}\right) \hat{\otimes} \Omega^{\bullet}\left(\Delta_{1}\right)
$$


which connects $P_{m}$ to $P_{m+1}$ and such that

$$
\xi_{m-1} \in \mathcal{F}_{m-1} \operatorname{Hom}\left(\operatorname{Ger}^{\vee}\left(V_{A}\right), C^{\bullet}\right)
$$

and

$$
P_{m}^{\prime}(t)-P_{m} \in \mathcal{F}_{m} \operatorname{Hom}\left(\operatorname{Ger}^{\vee}\left(V_{A}\right), C^{\bullet}\right) \hat{\otimes} \mathbb{K}[t]
$$

Let us now prove that one can construct a 1-cell

$$
P_{n+1}^{\prime}(t)+d t \xi_{n} \in \operatorname{Hom}\left(\operatorname{Ger}^{\vee}\left(V_{A}\right), C^{\bullet}\right) \hat{\otimes} \Omega^{\bullet}\left(\Delta_{1}\right)
$$

such that

$$
\begin{aligned}
& \left.P_{n+1}^{\prime}(t)\right|_{t=0}=P_{n+1}, \\
& \xi_{n} \in \mathcal{F}_{n} \operatorname{Hom}\left(\operatorname{Ger}^{\vee}\left(V_{A}\right), C^{\bullet}\right), \\
& P_{n+1}^{\prime}(t)-P_{n+1} \in \mathcal{F}_{n+1} \operatorname{Hom}\left(\operatorname{Ger}^{\vee}\left(V_{A}\right), C^{\bullet}\right) \hat{\otimes} \mathbb{K}[t],
\end{aligned}
$$

and the Maurer-Cartan element

$$
P_{n+2}:=\left.P_{n+1}^{\prime}(t)\right|_{t=1}
$$

satisfies the condition

$$
\widetilde{P}-P_{n+2} \in \mathcal{F}_{n+2} \operatorname{Hom}\left(\operatorname{Ger}^{\vee}\left(V_{A}\right), C^{\bullet}\right)
$$

Let us denote the difference $\widetilde{P}-P_{n+1}$ by $K$. Since $\widetilde{P}-P_{n+1} \in \mathcal{F}_{n+1}$ Hom $\left(\operatorname{Ger}^{\vee}\left(V_{A}\right), C^{\bullet}\right)$,

$$
K=\sum_{m \geq n+1} K_{m}, \quad K_{m} \in \operatorname{Hom}_{S_{m}}\left(\operatorname{Ger}^{\vee}(m) \otimes V_{A}^{\otimes m}, C^{\bullet}\right) .
$$

Subtracting the left hand side of the Maurer-Cartan equation

$$
\mathcal{D}\left(P_{n+1}\right)+\sum_{m \geq 2} \frac{1}{m !}\left\{P_{n+1}, P_{n+1}, \ldots, P_{n+1}\right\}_{m}=0
$$

from the left hand side of the Maurer-Cartan equation

$$
\mathcal{D}(\widetilde{P})+\sum_{m \geq 2} \frac{1}{m !}\{\widetilde{P}, \widetilde{P}, \ldots, \widetilde{P}\}_{m}=0
$$


we see that element (6.67) satisfies the equation

$$
\mathcal{D}(K)+\sum_{m \geq 1} \frac{1}{m !}\left\{P_{n+1}, \ldots, P_{n+1}, K\right\}_{m+1}+\sum_{m \geq 2} \frac{1}{m !}\{K, K, \ldots, K\}_{m}^{P_{n+1}}=0
$$

where the multi-bracket $\{K, K, \ldots, K\}_{m}^{P_{n+1}}$ is defined by the formula

$$
\left\{X_{1}, X_{2}, \ldots, X_{m}\right\}_{m}^{P_{n+1}}:=\sum_{q \geq 0} \frac{1}{q !}\left\{P_{n+1}, \ldots, P_{n+1}, X_{1}, X_{2}, \ldots, X_{m}\right\}_{q+m}
$$

Evaluating (6.70) on $\operatorname{Ger}^{\vee}(n+1) \otimes V_{A}^{\otimes(n+1)}$ and using the fact that

$$
K \in \mathcal{F}_{n+1} \operatorname{Hom}\left(\operatorname{Ger}^{\vee}\left(V_{A}\right), C^{\bullet}\right),
$$

we conclude that

$$
\mathfrak{d} \circ K_{n+1}=0
$$

where $\mathfrak{d}$ is the differential on $C^{\bullet}$.

Hence there exist elements

$$
K_{n+1}^{V_{A}} \in \operatorname{Hom}_{S_{n+1}}\left(\operatorname{Ger}^{\vee}(n+1) \otimes V_{A}^{\otimes(n+1)}, V_{A}\right)
$$

and

$$
K_{n+1}^{\prime} \in \operatorname{Hom}_{S_{n+1}}\left(\operatorname{Ger}^{\vee}(n+1) \otimes V_{A}^{\otimes(n+1)}, C^{\bullet}\right)
$$

such that

$$
K_{n+1}=I \circ K_{n+1}^{V_{A}}+\mathfrak{d} \circ K_{n+1}^{\prime}
$$

Next, evaluating (6.70) on $Y \in \operatorname{Ger}^{\vee}(n+2) \otimes V_{A}^{\otimes(n+2)}$ and using inclusion (6.72) again, we get the following identity

$$
\mathfrak{d} \circ K_{n+2}(Y)-K_{n+1} \circ Q_{\wedge,\{,\}}(Y)+\left\{P_{n+1}, K_{n+1}\right\}_{2}(Y)=0 .
$$

Unfolding $\left\{P_{n+1}, K_{n+1}\right\}_{2}(Y)$ we get

$$
\left\{P_{n+1}, K_{n+1}\right\}_{2}(Y)=\sum_{i=1}^{n+2} Q_{C} \cdot\left(\left(\operatorname{id}_{\mathrm{Ger}^{\vee}(2)} \otimes K_{n+1} \otimes I\right) \circ\left(\Delta_{\mathbf{t}_{i}} \otimes \mathrm{id}^{\otimes(n+2)}\right)(Y)\right),
$$


Fig. 6 The $(n+2)$-labeled planar tree $\mathbf{t}_{i}$

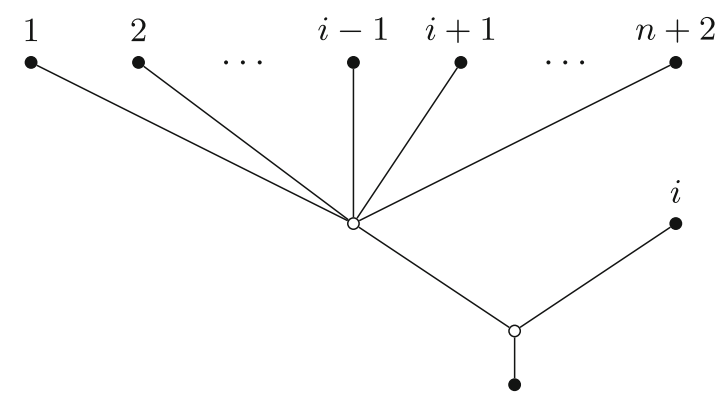

where $Q_{C} \bullet$ is the $\operatorname{Ger}_{\infty}$-structure on $C^{\bullet}, \mathbf{t}_{i}$ is the $(n+2)$-labeled planar tree shown on Fig. 6, and $\Delta_{\mathbf{t}_{i}}$ is the corresponding component of the comultiplication

$$
\Delta_{\mathbf{t}_{i}}: \operatorname{Ger}^{\vee}(n+2) \rightarrow \operatorname{Ger}^{\vee}(2) \otimes \operatorname{Ger}^{\vee}(n+1) .
$$

Now using (6.74) and (6.76), we rewrite (6.75) as follows

$$
\begin{aligned}
\mathfrak{d} \circ K_{n+2}(Y)-I \circ\left(K_{n+1}^{V_{A}} \bullet \alpha\right)(Y) \\
+\sum_{i=1}^{n+2} Q_{C} \cdot\left(\left(\mathrm{id}_{\operatorname{Ger}^{\vee}(2)} \otimes\left(\mathfrak{d} \circ K_{n+1}^{\prime}\right) \otimes I\right) \circ\left(\Delta_{\mathbf{t}_{i}} \otimes \mathrm{id}^{\otimes(n+2)}\right)(Y)\right) \\
+\sum_{i=1}^{n+2} Q_{C} \cdot\left(\left(\operatorname{id}_{\mathrm{Ger}^{\vee}(2)} \otimes\left(I \circ K_{n+1}^{V_{A}}\right) \otimes I\right) \circ\left(\Delta_{\mathbf{t}_{i}} \otimes \mathrm{id}^{\otimes(n+2)}\right)(Y)\right)=0,
\end{aligned}
$$

where $\alpha$ is defined in (6.2).

Since the last two sums in (6.78) involve only binary $\operatorname{Ger}_{\infty}$-operations on $C^{\bullet}$ and these binary operations induce the usual multiplication and the Schouten bracket on $V_{A}$, we conclude that each term in the first sum in (6.78) is $\mathfrak{d}$-exact and the second sum in (6.78) is cohomologous to

$$
I \circ\left(\alpha \bullet K_{n+1}^{V_{A}}\right)(Y)
$$

Therefore, identity (6.78) implies that for every $Y \in \operatorname{Ger}^{\vee}(n+2) \otimes V_{A}^{\otimes(n+2)}$ the expression

$$
I \circ\left(\alpha \bullet K_{n+1}^{V_{A}}-K_{n+1}^{V_{A}} \bullet \alpha\right)(Y)
$$

is $\mathfrak{d}$-exact. Thus

$$
\alpha \bullet K_{n+1}^{V_{A}}-K_{n+1}^{V_{A}} \bullet \alpha=0
$$


or, in other words, the element $K_{n+1}^{V_{A}}$ is a cocycle in complex (6.3) with differential (6.5).

Hence, by Theorem 6.1, there exists a degree -1 element

$$
\widetilde{K}_{n}^{V_{A}} \in \operatorname{Hom}_{S_{n}}\left(\operatorname{Ger}^{\vee}(n) \otimes V_{A}^{\otimes(n)}, V_{A}\right)
$$

such that

$$
K_{n+1}^{V_{A}}=\left[\alpha, \widetilde{K}_{n}^{V_{A}}\right]
$$

Let us now consider the degree -1 element

$$
\xi_{n}=I \circ \widetilde{K}_{n}^{V_{A}}+K_{n+1}^{\prime \prime} \in \mathcal{F}_{n} \operatorname{Hom}\left(\operatorname{Ger}^{\vee}\left(V_{A}\right), C^{\bullet}\right),
$$

where $\widetilde{K}_{n}^{V_{A}}$ is element (6.79) entering Eq. (6.80) and $K_{n+1}^{\prime \prime}$ is an element in

$$
\operatorname{Hom}_{S_{n+1}}\left(\operatorname{Ger}^{\vee}(n+1) \otimes V_{A}^{\otimes(n+1)}, C^{\bullet}\right)
$$

which will be determined later.

Using $\xi_{n}$, we define $P_{n+1}^{\prime}(t) \in \operatorname{Hom}\left(\operatorname{Ger}^{\vee}\left(V_{A}\right), C^{\bullet}\right) \hat{\otimes} \mathbb{K}[t]$ as the limiting element of the recursive procedure

$$
\begin{aligned}
\left(P^{\prime}\right)^{(0)} & :=P_{n+1} \\
\left(P^{\prime}\right)^{(k+1)}(t) & :=P_{n+1}+\int_{0}^{t} d t_{1}\left(\mathcal{D}\left(\xi_{n}\right)+\sum_{m \geq 1} \frac{1}{m !}\left\{\left(P^{\prime}\right)^{(k)}\left(t_{1}\right), \ldots,\left(P^{\prime}\right)^{(k)}\left(t_{1}\right), \xi_{n}\right\}_{m+1}\right) .
\end{aligned}
$$

Since

$$
\mathfrak{d}\left(I \circ \widetilde{K}_{n}^{V_{A}}\right)=0
$$

the element $\xi_{n}$ satisfies the condition

$$
\mathcal{D}\left(\xi_{n}\right) \in \mathcal{F}_{n+1} \operatorname{Hom}\left(\operatorname{Ger}^{\vee}\left(V_{A}\right), C^{\bullet}\right) .
$$

Hence, by Lemma 5.1, the sum

$$
P_{n+1}^{\prime}(t)+d t \xi_{n} \in \operatorname{Hom}\left(\operatorname{Ger}^{\vee}\left(V_{A}\right), C^{\bullet}\right) \hat{\otimes} \Omega^{\bullet}\left(\Delta_{1}\right)
$$

is a 1-cell in the $\infty$-groupoid corresponding to $\operatorname{Hom}\left(\operatorname{Ger}^{\vee}\left(V_{A}\right), C^{\bullet}\right)$ satisfying (6.64) and such that the Maurer-Cartan element $P_{n+2}(6.65)$ satisfies the condition

$$
P_{n+2}-P_{n+1}-\mathcal{D}\left(\xi_{n}\right)-\left\{P_{n+1}, \xi_{n}\right\}_{2} \in \mathcal{F}_{n+2} \operatorname{Hom}\left(\operatorname{Ger}^{\vee}\left(V_{A}\right), C^{\bullet}\right)
$$


Fig. 7 The $(n+1)$-labeled planar tree $\mathbf{t}_{i}^{\prime}$

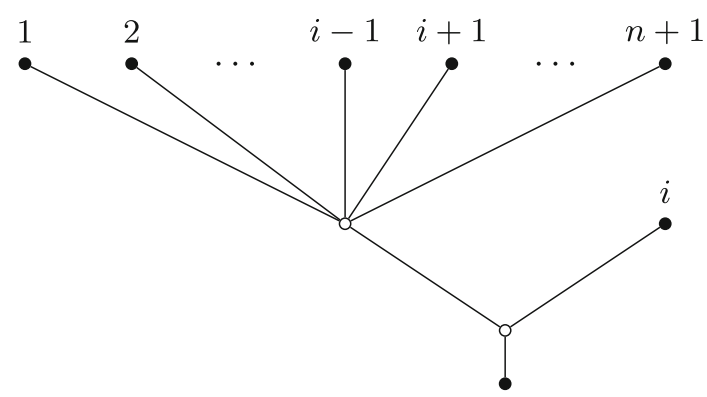

Let us now show that, by choosing the element $K_{n+1}^{\prime \prime}$ in (6.81) appropriately, we can get desired inclusion (6.66).

For this purpose we unfold $\left\{P_{n+1}, \xi_{n}\right\}_{2}(Y)$ for an arbitrary $Y \in \operatorname{Ger}^{\vee}(n+1) \otimes$ $V_{A}^{\otimes(n+1)}$ and get

$\left\{P_{n+1}, \xi_{n}\right\}_{2}(Y)=\sum_{i=1}^{n+1} Q_{C} \cdot\left(\left(\operatorname{id}_{\operatorname{Ger}^{\vee}(2)} \otimes\left(I \circ \widetilde{K}_{n}^{V_{A}}\right) \otimes I\right) \circ\left(\Delta_{\mathbf{t}_{i}^{\prime}} \otimes \mathrm{id}^{\otimes(n+1)}\right)(Y)\right)$,

where $Q_{C} \bullet$ is the $\operatorname{Ger}_{\infty}$-structure on $C^{\bullet}, \mathbf{t}_{i}^{\prime}$ is the $(n+1)$-labeled planar tree shown on Fig. 7, and $\Delta_{\mathbf{t}_{i}^{\prime}}$ is the corresponding component of the comultiplication

$$
\Delta_{\mathbf{t}_{i}^{\prime}}: \operatorname{Ger}^{\vee}(n+1) \rightarrow \operatorname{Ger}^{\vee}(2) \otimes \operatorname{Ger}^{\vee}(n) .
$$

Since the right hand side of (6.85) involves only binary $\operatorname{Ger}_{\infty}$-operations on $C^{\bullet}$ and these binary operations induce the usual multiplication and the Schouten bracket on $V_{A}$, we conclude that $\left\{P_{n+1}, \xi_{n}\right\}_{2}(Y)$ is cohomologous (in $C^{\bullet}$ ) to

$$
I \circ\left(\alpha \bullet \widetilde{K}_{n}^{V_{A}}\right)(Y),
$$

where $\alpha$ is defined in (6.2).

In other words, there exists an element

$$
\phi \in \operatorname{Hom}_{S_{n+1}}\left(\operatorname{Ger}^{\vee}(n+1) \otimes V_{A}^{\otimes(n+1)}, C^{\bullet}\right)
$$

such that

$$
\left\{P_{n+1}, \xi_{n}\right\}_{2}(Y)=I \circ\left(\alpha \bullet \widetilde{K}_{n}^{V_{A}}\right)(Y)+\mathfrak{d} \circ \phi(Y) .
$$

Hence the expression $\left(\mathcal{D}\left(\xi_{n}\right)+\left\{P_{n+1}, \xi_{n}\right\}_{2}\right)(Y)$ can be rewritten as

$$
\left(\mathcal{D}\left(\xi_{n}\right)+\left\{P_{n+1}, \xi_{n}\right\}_{2}\right)(Y)=\mathfrak{d} \circ K_{n+1}^{\prime \prime}(Y)+\mathfrak{d} \circ \phi(Y)+I \circ\left[\alpha, \widetilde{K}_{n}^{V_{A}}\right](Y) .
$$


Thus if

$$
K_{n+1}^{\prime \prime}=K_{n+1}^{\prime}-\phi
$$

then Eqs. (6.74), (6.80), and inclusion (6.84) imply that (6.66) holds, as desired.

Thus we showed that one can construct an infinite sequence of Maurer-Cartan elements

$$
P=P_{2}, P_{3}, P_{4}, \ldots
$$

and an infinite sequence of 1-cells $(m \geq 2)$

$$
P_{m}^{\prime}(t)+d t \xi_{m-1} \in \operatorname{Hom}\left(\operatorname{Ger}^{\vee}\left(V_{A}\right), C^{\bullet}\right) \hat{\otimes} \Omega^{\bullet}\left(\Delta_{1}\right)
$$

such that for every $m \geq 2$

$$
\widetilde{P}-P_{m} \in \mathcal{F}_{m} \operatorname{Hom}\left(\operatorname{Ger}^{\vee}\left(V_{A}\right), C^{\bullet}\right),
$$

the 1-cell $P_{m}^{\prime}(t)+d t \xi_{m-1}$ connects $P_{m}$ to $P_{m+1}$

$$
\xi_{m-1} \in \mathcal{F}_{m-1} \operatorname{Hom}\left(\operatorname{Ger}^{\vee}\left(V_{A}\right), C^{\bullet}\right),
$$

and

$$
P_{m}^{\prime}(t)-P_{m} \in \mathcal{F}_{m} \operatorname{Hom}\left(\operatorname{Ger}^{\vee}\left(V_{A}\right), C^{\bullet}\right) \hat{\otimes} \mathbb{K}[t]
$$

Since the $\Lambda^{-1}$ Lie $_{\infty}$-algebra $\operatorname{Hom}\left(\operatorname{Ger}^{\vee}\left(V_{A}\right), C^{\bullet}\right)$ is complete with respect to "arity" filtration (5.29), inclusions (6.90) and (6.91) imply that we can form the infinite composition $^{21}$ of all 1-cells (6.89) and get a 1-cell which connects the Maurer-Cartan element $P=P_{2}$ to the Maurer-Cartan element $\widetilde{P}$.

Corollary 6.4 is proved.

\section{Appendix C: On derivations of $\mathrm{Cyl}\left(\Lambda^{2} \operatorname{coCom}\right)$}

Let $\mathcal{C}$ be a coaugmented cooperad in the category of graded vector spaces and $\mathcal{C}$ 。 be the cokernel of the coaugmentation. As above, we assume that $\mathcal{C}(0)=\mathbf{0}$ and $\mathcal{C}(1)=\mathbb{K}$.

Following [22, Section 3], [13], we will denote by $\mathrm{Cyl}(\mathcal{C})$ the 2-colored dg operad whose algebras are pairs $(V, W)$ with the data

1. a $\operatorname{Cobar}(\mathcal{C})$-algebra structure on $V$,

2. a Cobar $(\mathcal{C})$-algebra structure on $W$, and

3. an $\infty$-morphism $F$ from $V$ to $W$, i.e. a homomorphism of corresponding $\operatorname{dg} \mathcal{C}$ coalgebras $\mathcal{C}(V) \rightarrow \mathcal{C}(W)$.

\footnotetext{
${ }^{21}$ Note that the composition of 1-cells in an infinity groupoid is not unique but this does not create a problem.
} 
In fact, if we forget about the differential, then $\operatorname{Cyl}(\mathcal{C})$ is a free operad on a certain 2-colored collection $\mathcal{M}(\mathcal{C})$ naturally associated to $\mathcal{C}$.

Following the conventions of Sect. 3, we denote by

$$
\operatorname{Der}^{\prime}(\operatorname{Cyl}(\mathcal{C}))
$$

the $\mathrm{dg}$ Lie algebra of derivations $\mathcal{D}$ of $\operatorname{Cyl}(\mathcal{C})$ subject to the condition

$$
p \circ \mathcal{D}=0
$$

where $p$ is the canonical projection from $\operatorname{Cyl}(\mathcal{C})$ onto $\mathcal{M}(\mathcal{C})$.

We have the following generalization of (3.3):

Proposition 7.1 The dg Lie algebra $\operatorname{Der}^{\prime}\left(\operatorname{Cyl}\left(\Lambda^{2} \operatorname{coCom}\right)\right)$ does not have non-zero elements in degrees $\leq 0$, i.e.

$$
\operatorname{Der}^{\prime}\left(\operatorname{Cyl}\left(\Lambda^{2} \operatorname{coCom}\right)\right)^{\leq 0}=\mathbf{0} \text {. }
$$

Proof Let us denote by $\alpha$ and $\beta$, respectively, the first and the second color for the collection $\mathcal{M}\left(\Lambda^{2}\right.$ coCom) and the operad $\operatorname{Cyl}\left(\Lambda^{2} \operatorname{coCom}\right)$.

Recall from [22] that $\operatorname{Cyl}\left(\Lambda^{2}\right.$ coCom $)$ is generated by the collection $\mathcal{M}=$ $\mathcal{M}\left(\Lambda^{2}\right.$ coCom) with

$$
\begin{aligned}
& \mathcal{M}(n, 0 ; \alpha)=\mathbf{s} \Lambda^{2} \operatorname{coCom}_{\circ}(n)=\mathbf{s}^{3-2 n} \mathbb{K}, \\
& \mathcal{M}(0, n ; \beta)=\mathbf{s} \Lambda^{2} \operatorname{coCom}_{\circ}(n)=\mathbf{s}^{3-2 n} \mathbb{K}, \\
& \mathcal{M}(n, 0 ; \beta)=\Lambda^{2} \operatorname{coCom}(n)=\mathbf{s}^{2-2 n} \mathbb{K},
\end{aligned}
$$

and with all the remaining spaces being zero. Let $\mathcal{D}$ be a derivation of $\operatorname{Cyl}\left(\Lambda^{2} \operatorname{coCom}\right)$ of degree $\leq 0$.

Since

$$
\begin{aligned}
& \text { Cyl }\left(\Lambda^{2} \operatorname{coCom}\right)(n, 0, \alpha)=\Lambda \operatorname{Lie}_{\infty}(n) \text { and } \operatorname{Cyl}\left(\Lambda^{2} \operatorname{coCom}\right)(0, n, \beta) \\
& =\Lambda \operatorname{Lie}_{\infty}(n),
\end{aligned}
$$

observation (3.3) implies that

$$
\left.\mathcal{D}\right|_{\mathcal{M}(n, 0 ; \alpha)}=\left.\mathcal{D}\right|_{\mathcal{M}(0, n ; \beta)}=0
$$

Hence, it suffices to show that

$$
\left.\mathcal{D}\right|_{\mathcal{M}(n, 0 ; \beta)}=0
$$

Let us denote by $\pi_{0}\left(\operatorname{Tree}_{k}(n)\right)$ the set of isomorphism classes of labeled 2-colored planar trees corresponding to corolla $(n, 0 ; \beta)$ with $k$ internal vertices. Figure 8 show 

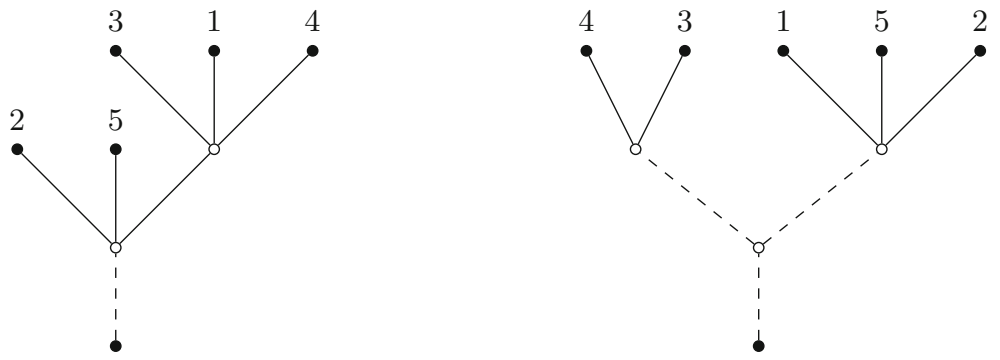

Fig. 8 Solid edges carry the color $\alpha$ and dashed edges carry the color $\beta$; internal vertices are denoted by small white circles; leaves and the root vertex are denoted by small black circles

two examples of such trees with $n=5$ leaves. The left tree has $k=2$ internal vertices and the right tree has $k=3$ internal vertices.

For a generator $X \in \mathcal{M}(n, 0 ; \beta)=\mathbf{s}^{2-2 n} \mathbb{K}$, the element $\mathcal{D}(X) \in \operatorname{Cyl}\left(\Lambda^{2} \operatorname{coCom}\right)$ takes the form

$$
\mathcal{D}(X)=\sum_{k \geq 2} \sum_{z \in \pi_{0}\left(\operatorname{Tree}_{k}(n)\right)}\left(\mathbf{t}_{z} ; X_{1}, \ldots, X_{k}\right)
$$

where $\mathbf{t}_{z}$ is a representative of an isomorphism class $z \in \pi_{0}\left(\operatorname{Tree}_{k}(n)\right)$ and $X_{i}$ are the corresponding elements of $\mathcal{M}$.

For every term in sum (7.4), we have $k_{1} X_{i}$ 's in $\mathbf{s} \Lambda^{2}$ coCom。 (call them $X_{i_{a}}$ ), and $k_{2} X_{i}$ 's in $\Lambda^{2}$ coCom (call them $X_{j_{b}}$ ).

We obviously have that $k=k_{1}+k_{2}$ and

$$
|\mathcal{D}|=\sum_{a=1}^{k_{1}}\left|X_{i_{a}}\right|+\sum_{b=1}^{k_{2}}\left|X_{j_{b}}\right|-|X|
$$

or equivalently

$$
|\mathcal{D}|=2(n-1)+\sum_{a=1}^{k_{1}}\left(3-2 n_{i_{a}}\right)+\sum_{b=1}^{k_{2}}\left(2-2 n_{j_{b}}\right),
$$

where $n_{i_{a}}$ (resp. $n_{j_{b}}$ ) is the number of incoming edges of the vertex corresponding to $X_{i_{a}}\left(\operatorname{resp} . X_{j_{b}}\right)$.

On the other hand, a simple combinatorics of trees shows that

$$
n-1=\sum_{a=1}^{k_{1}}\left(n_{i_{a}}-1\right)+\sum_{b=1}^{k_{2}}\left(n_{j_{b}}-1\right)
$$

and hence

$$
|\mathcal{D}|=k_{1}
$$


Since $|\mathcal{D}| \leq 0$ the latter is possible only if $k_{1}=0=|\mathcal{D}|$, i.e. every tree in the sum $\mathcal{D}(X)$ is assembled exclusively from mixed colored corollas. That would force every tree $\mathbf{t}$ to have only one internal vertex which contradicts to the fact that the summation in (7.4) starts at $k=2$.

Therefore (7.3) holds and the proposition follows.

\section{References}

1. Alekseev, A., Torossian, C.: The Kashiwara-Vergne conjecture and Drinfeld's associators. Ann. Math. (2) 175(2), 415-463 (2012). arXiv:0802.4300

2. Bar-Natan, D.: On associators and the Grothendieck-Teichmueller group. I Selecta Math. (N.S.) 4(2), 183-212 (1998)

3. Batanin, M., Markl, M.: Crossed interval groups and operations on the Hochschild cohomology. J. Noncommut. Geom. 8(3), 655-693 (2014). arXiv:0803.2249

4. Berger, C., Fresse, B.: Combinatorial operad actions on cochains. Math. Proc. Camb. Philos. Soc. 137(1), 135-174 (2004)

5. Dolgushev, V.A., Rogers, C.L.: Notes on algebraic operads, graph complexes, and Willwacher's construction, mathematical aspects of quantization. In: Contemp. Math., vol. 583, pp. 25-145. AMS, Providence (2012). arXiv:1202.2937

6. Dolgushev, V.A., Rogers, C.L.: On an enhancement of the category of shifted L-infinity algebras. arXiv: 1406.1744

7. Dolgushev, V.A., Hoffnung, A.E., Rogers, C.L.: What do homotopy algebras form? Adv. Math. 274, 562-605 (2015). arXiv:1406.1751

8. Dolgushev, V.A., Tamarkin, D.E., Tsygan, B.L.: Proof of swiss cheese version of Deligne's conjecture. Int. Math. Res. Notices 2011, 4666-4746 (2011)

9. Dolgushev, V.A., Willwacher, T.H.: Operadic Twisting — with an application to Deligne's conjecture. J. Pure Appl. Algebra 219(5), 1349-1428 (2015). arXiv:1207.2180

10. Dotsenko, V., Poncin, N.: A tale of three homotopies. arXiv:1208.4695, Version 2

11. Drinfeld, V.G.: On quasitriangular quasi-Hopf algebras and on a group that is closely connected with $\operatorname{Gal}(\overline{\mathbb{Q}} / \mathbb{Q})$. Algebra i Analiz. 2(4), 149-181 (1990) (Russian) (translation in Leningrad Math. J. 2(4), 829-860, 1991)

12. Fiedorowicz, Z.: The symmetric bar construction. https://people.math.osu.edu/fiedorowicz.1/. Accessed 21 June 2015

13. Fresse, B.: Operadic cobar constructions, cylinder objects and homotopy morphisms of algebras over operads, Alpine perspectives on algebraic topology. In: Contemp. Math., vol. 504, pp. 125-188. Am. Math. Soc., Providence (2009)

14. Getzler, E.: Lie theory for nilpotent $L_{\infty}$-algebras. Ann. Math. (2) 170(1), 271-301 (2009). arXiv:math/0404003

15. Hinich, V.: Tamarkin's proof of Kontsevich formality theorem. Forum Math. 15(4), 591-614 (2003). arXiv:math.QA/0003052

16. Hochschild, G., Kostant, B., Rosenberg, A.: Differential forms on regular affine algebras. Trans. Am. Math. Soc. 102, 383-408 (1962)

17. Kontsevich, M., Soibelman, Y.: Deformations of algebras over operads and the Deligne conjecture. In: Proceedings of the Moshé Flato Conference Math. Phys. Stud. vol. 21, pp. 255-307, Kluwer, Dordrecht (2000)

18. Kontsevich, M.: Operads and motives in deformation quantization. Lett. Math. Phys. 48(1), 35-72 (1999)

19. Loday, J.-L.: Cyclic homology. In: Grundlehren der Mathematischen Wissenschaften (Appendix E by Maria O. Ronco), vol. 301. Springer, Berlin (1992)

20. Loday, J.-L., Vallette, B.: Algebraic operads. In: Grundlehren der Mathematischen Wissenschaften, vol. 346, pp. xxiv+634. Springer, Heidelberg (2012)

21. McClure, J.E., Smith, J.H.: A solution of Deligne's Hochschild cohomology conjecture. Contemp. Math. 293, 153-193 (2002) (Am. Math. Soc., Providence). arXiv:math.QA/9910126

22. Paljug, B.: Action of derived automorphisms on infinity morphisms. JHRS (to appear) (2015). arXiv: 1305.4699 
23. Tamarkin, D.: What do DG categories form? Compos. Math. 143(5), 1335-1358 (2007). arXiv:math.CT/0606553

24. Tamarkin, D.E.: Another proof of M. Kontsevich formality theorem (1998). arXiv:math/9803025

25. Tamarkin, D.: Formality of chain operad of little discs. Lett. Math. Phys. 66(1-2), 65-72 (2003)

26. Voronov, A.A.: Homotopy Gerstenhaber algebras. In: Proceedings of the Moshé Flato Conference Math. Phys. Stud., vol. 22, pp. 307-331. Kluwer, Dordrecht (2000)

27. Willwacher, T.: M. Kontsevich's graph complex and the Grothendieck-Teichmueller Lie algebra, Invent. Math. 200(3), 671-760 (2015) 